Revue des patrimoines

40 | 2019

Lits historiques. Première anthologie des lits européens du XVe au XIXe siècle

\title{
Les lits royaux de la Renaissance à la lumière des archives de Louis XIV
}

Royal Beds of the Renaissance as seen in the Archives of Louis XIV

\section{Jean Vittet}

\section{(2) OpenEdition}

\section{Journals}

Édition électronique

URL : http://journals.openedition.org/insitu/22950

DOI : $10.4000 /$ insitu. 22950

ISSN : 1630-7305

Éditeur

Ministère de la Culture

Référence électronique

Jean Vittet, "Les lits royaux de la Renaissance à la lumière des archives de Louis XIV », In Situ [En

ligne], 40 | 2019, mis en ligne le 19 septembre 2019, consulté le 14 novembre 2019. URL : http://

journals.openedition.org/insitu/22950; DOI : 10.4000/insitu.22950

Ce document a été généré automatiquement le 14 novembre 2019.

\section{cc) () $९$}

In Situ Revues des patrimoines est mis à disposition selon les termes de la licence Creative Commons Attribution - Pas d'Utilisation Commerciale - Pas de Modification 4.0 International. 


\title{
Les lits royaux de la Renaissance à la lumière des archives de Louis XIV
}

Royal Beds of the Renaissance as seen in the Archives of Louis XIV

\author{
Jean Vittet
}

Remerciements : Françoise Aujogue, Muriel Barbier, Hélène Cavalié, Nicolas Courtin, Roselyne Laurieux.

1 En matière de lits, les collections royales françaises possédaient à la Renaissance des exemplaires exceptionnels mais en raison du caractère lacunaire des comptes et des inventaires, il est souvent difficile d'en avoir une connaissance satisfaisante ${ }^{1}$. Devant ce manque d'informations, il peut être profitable de s'appuyer sur des documents descriptifs rédigés postérieurement, lorsque les œuvres existaient encore. De ce point de vue, l'« Inventaire général du mobilier de la Couronne sous Louis XIV (1663-1715) », publié par Jules Guiffrey en 1885-1886, qui décrit un certain nombre de lits ou de dais de la Renaissance, peut s'avérer d'une grande utilité2.

\section{Considérations liminaires sur l'inventaire de Louis XIV}

2 Toutefois, pour toute recherche sur les collections de Louis XIV, la question se pose de savoir si l'« Inventaire général » en six volumes (quatre subsistent), clos primitivement en 1673, ne concerne que le fonds conservé au Garde-Meuble à Paris ou couvre l'ensemble des collections, indépendamment de leur localisation. Guiffrey n'apporte aucun éclairage sur ce point essentiel. Or l'analyse du document original permet de répondre en partie à cette interrogation ${ }^{3}$. Le document, fâcheusement allégé par Guiffrey pour la publication, porte en fait le titre d'« Inventaire général des meubles de la Couronne et des Maisons royalles» et comporte de nombreuses indications de localisation ou de provenance, non reprises lors du passage à l'impression. Considéré dans sa formation initiale de 1673, sans les enrichissements postérieurs, l'inventaire ne concerne pratiquement que trois fonds: le Garde-Meuble à Paris, Versailles et Vincennes ${ }^{4}$. D'autre part, dans le classement originel, les chapitres des «Lits et 
emmeublemens » et des «Daiz », où sont décrits les lits de la Renaissance, se trouvaient en tête du volume II de l'inventaire. Les lits et dais royaux étaient les éléments d'ameublement les plus sacrés, notamment parce qu'ils avaient été brodés par des reines. Ils étaient en outre des meubles essentiels dans l'exercice du pouvoir, aussi importants que les trônes. Présents dans les cérémonies de baptêmes, de mariages et de pompes funèbres, ils symbolisaient la continuité dynastique. Sur l'inventaire, on les avait donc placés avant les tapisseries de lisse historiées, quand Guiffrey les relègue, comme quantité négligeable, à la fin de son second volume.

Outre cet inventaire de 1673, qui est le plus important, on dispose d'un inventaire antérieur, remontant à 1666, qui ne comprend que le fonds parisien du Garde-Meuble. Il ne concerne que quelques meubles brodés mais les descriptions en sont très détaillées ${ }^{5}$. On bénéficie encore de l'Inventaire général dit de 1729 , mais rédigé dès 1717-1718, qui complète les descriptions, notamment pour ce qui concerne les dimensions, et donne parfois d'utiles indications héraldiques ${ }^{6}$.

4 Pour cette publication, nous avons pris le parti de retranscrire le texte intégral des descriptions de lits et dais de la Renaissance de l'Inventaire général de 1673, d'après le document original, souvent abrégées par Guiffrey, en les faisant suivre de leur historique tel qu'il peut être reconstitué?.

\section{Les « Lits et emmeublemens » et les « Daiz »}

5 Dans l'inventaire de 1673, les lits de la Renaissance sont répartis entre deux sections en tête du volume II. Au chapitre des «Lits et emmeublemens ", on en trouve environ dixhuit qui paraissent relever de cette période, leur emblématique ou leur historique permettant de les relier aux souverains de l'époque. Trois peuvent ainsi être rattachés à François I ${ }^{\text {er }}$, un à Henry VIII d'Angleterre, deux aux Bourbons, un aux rois de Navarre, quatre à la reine de Navarre Jeanne d'Albret (1528-1572), un à Henri IV qui a fait porter à Fontainebleau les lits brodés des rois de Navarre en 1602 et un à Diane de Poitiers. Quatre sont d'origine inconnue, tandis qu'un dernier provient de Richelieu. Nous l'avons comptabilisé ici car il a parfois été daté du xvie siècle. L'absence de localisation spécifiée sur l'inventaire jusqu'au $\mathrm{n}^{\circ} 46$ montre que les lits jusqu'à ce numéro étaient conservés à Paris, à l'hôtel du Petit-Bourbon. À quelques exceptions près, tous les lits de la Renaissance sont regroupés dans cette section. $\mathrm{Du} \mathrm{n}^{\circ} 47$ au $\mathrm{n}^{\circ} 57$, l'annotation marginale («Am.») indique en revanche que les meubles proviennent du château d'Amboise, mais nous ne les avons pas retenus dans notre comptage car ils semblent postérieurs au XVI ${ }^{e}$ siècle, comme le laisse penser leur association à de nombreux sièges. À partir du $n^{\circ} 58$ jusqu'au $n^{\circ} 85$, l'annotation («Vin.») fournit l'indication d'une extraction du château de Vincennes.

6 Au chapitre des « Dais », on peut repérer vingt-cinq articles environ relevant de notre sujet, mais en fait quatre, voire huit d'entre eux, ont déjà été cités parmi les ensembles inventoriés au chapitre des «Lits et emmeublemens». L'absence d'annotation de provenance en marge laisse penser qu'ils étaient également conservés à Paris. Trois dais proviennent de François $\mathrm{I}^{\mathrm{er}}$, un de Henri II, deux des ducs de Guise, deux des Bourbons, cinq des rois de Navarre, trois de Jeanne d'Albret, un de Henri IV, cinq sont d'origine inconnue. Un dernier venant de Richelieu faisait partie du meuble correspondant au chapitre des Lits. 


\section{Des transformations radicales pour Saint-Germain-en- Laye en 1689}

7 Lorsqu'on avance beaucoup plus loin dans le classement des « Lits et emmeublemens ", on constate que certains lits ou dais, déjà inscrits en tête du chapitre, se retrouvent à nouveau, mais décrits un peu différemment et pourvus de nouveaux numéros $\left(\mathrm{n}^{\circ}\right.$ 1380-1386) ${ }^{8}$. Cela s'explique par le fait que ces ensembles ont été retravaillés en 1687-1689 afin de garnir l'appartement du roi au château de Saint-Germain-en-Laye, projet laissé inachevé à la demande de Louis XIV9. Les nouvelles descriptions ont parfois été complétées, alors que les œuvres ont été notoirement modifiées par les tapissiers, notamment par Doublet, dont le nom est cité. Par exemple, dans la grande chambre du roi, le Garde-Meuble confectionne un lit à partir des anciens lits du Cerf fragile et de l'histoire de Joseph $\left(n^{\circ} 10\right.$ et 11$)$ et tend au mur une tenture de velours rouge tirée des dais aux Lions emparqués provenant des rois de Navarre $\left(n^{\circ} 10\right.$ et 12 des dais).

8 Pour la compréhension de ces changements, on dispose encore de deux mémoires inédits du brodeur Desprez, qui a restauré et transformé, parfois de manière radicale, une partie de ces lits brodés en $1689^{10}$. Pour le grand cabinet du roi, il a ainsi " accommodé» les Devises de Pau ( ${ }^{\circ} 1$ des Lits). Pour la petite chambre du roi, plusieurs ensembles ont été remployés, notamment le lit de l'Enlèvement d'Hélène, peu décrit dans l'Inventaire général ( $\left.\mathrm{n}^{\circ} 12\right)$. Grâce au mémoire du brodeur, on apprend que certaines scènes profanes ont été modifiées pour leur donner une connotation religieuse : ainsi l'Incendie de Troie est transformé en Embrasement de Sodome et Gomorrhe, et le Cheval d'airain remplacé par Loth et sa femme et leurs deux filles.

9 Il y avait donc assez peu de lits de la Renaissance à proprement parler dans les collections de Louis XIV - huit exactement, les autres ensembles se présentant plutôt sous forme de dais, voire de simples tentures. Par ailleurs, la majorité d'entre eux provenait de Henri IV et de ses ancêtres, tandis que ceux hérités des Valois étaient manifestement plus rares. Même avec les descriptions de l'Inventaire général, ces lits et dais restent difficiles à étudier en raison du manque de clarté des formulations et des difficultés de coordination avec les autres inventaires de collections. Peu d'identifications sont possibles, d'autant que la plupart ont été détruits dès les règnes de Louis XV et de Louis XVI, ce qui n'avait guère été soupçonné jusqu'à présent, car leur disparition avait presque toujours été imputée à la Révolution.

\section{Extrait de l'« Inventaire général des meubles de la Couronne et des maisons royalles ", tome second}

\section{Lits et emmeublemens ${ }^{11}$}

1. Un emmeublement de taillure d'or et d'argent par carreaux fonds rouge avec des chiffres d'or et octogones où il y a plusieurs devises d'or et d'argent et soye, appellé anciennement l'emmeublement de Pau, consistant en un lit complet, un tapis de table, un daix et une tapisserie, ledit lit composé de vingt-sept pièces, dont dix sont de la broderie cy-dessus, sçavoir trois pantes de dehors, trois sousbassemens, deux bonnes 
grâces et deux cantonnières, et les dix-sept autres pièces de satin vert naissant, parsemé de broderie légère par carreaux, fonds rouge, avec chiffres d'or comme dessus et octogones, dans lesquels il y a des bouquets de fleurs, sçavoir le fonds, dossier, trois pantes de dedans, couverture de parade, trois rideaux, quatre fourreaux de pilliers et quatre pommes avec leurs glands d'or et d'argent faux, les rideaux, bonnes grâces et cantonnières doublez de taffetas vert; trois matelas de satin rouge; un bois de lit de cinq pieds $1 / 2$ de large sur sept pieds $1 / 2$ de hault; le tapis de table à quatre pantes de mesme broderie que le lit; une couverture de taffetas picquée vert naissant; nota qu'il n'y a point de bois de table et qu'il y manque beaucoup de frange, tant au lit qu'au tapis; le daix composé du fonds de la queue et de sept pantes aussy de la mesme broderie du lit ; la tapisserie pareille à l'emmeublement appellé les devises de Pau, aux quatre coins de laquelle sont les armes de Bourbon et de Navarre, contenant 29 aunes $1 / 4$ de cours, sur 3 aunes $1 / 4$ de hault, en 9 pièces. [Annotation en marge :] Voyez le Journal du garde-meuble commancé le $19^{\mathrm{e}}$ aoust 1685 à fol. $128 \mathrm{v}^{\circ}$.

11 Historique : brodé par Jeanne d'Albret (1528-1572), reine de Navarre ${ }^{12}$; à Nérac dès $1569^{13}$; transporté de Pau à Nérac en $1578^{14}$; passé à Henri IV ; envoyé pour l'essentiel à Paris en 1597 et en $1601^{15}$; la tenture donnée à Gabrielle d'Estrées (1573-1599) par Henri IV dès $1597^{16}$, mais une confusion est possible avec le meuble $\mathrm{n}^{\circ} 89$ ci-dessous ; le fond du grand ciel porté à Fontainebleau en $1602^{17}$; la tenture employée en 1606 dans la chambre d'une fille d'Henri IV, la Petite Madame, pour son baptême à Fontainebleau ${ }^{18}$; employée à Reims en 1610 pour le sacre de Louis XIII ${ }^{19}$; au Garde-Meuble à Paris en $1666^{20}$; huit pièces employées au Palais-Royal en $1667^{21}$; transformé en 1689 pour être installé à Saint-Germain-en-Laye ${ }^{22} ; \mathrm{n}^{\circ} 1$ du chapitre des "Meubles de broderie sur toutes sortes de fonds » de l'inventaire de 1729 (le chiffre AL est mentionné) ${ }^{23}$; mis en vente en 1751 sans succès et détruit en $1784-1785^{24}$. Voir aussi le $\mathrm{n}^{0} 3$ ci-dessous avec lequel cet ensemble peut être confondu. Le dais correspondant se trouve au $\mathrm{n}^{\circ} 14 \mathrm{du}$ chapitre des dais.

12 2. Une tenture de tapisserie de petit point relevée d'or et de soye représentant des fables des Métamorphoses en trois pièces, composées chacune d'un tableau octogone dans le milieu et aux quatre coins de quatre ronds dans chacun desquels est la devise de la salemandre, le tout rapporté sur un fonds de velours rouge cramoisy avec des branches de lières et de lauriers liées ensemble, contenant treize aunes $1 / 2$ de cours sur 3 aunes $3 / 4$ de hault. [Annotation en marge :] Deschargé suivant le certifficat du $30^{\mathrm{e}}$ mars 1700 et porté au $\mathrm{n}^{\circ} 1383$ du présent inventaire. $\mathrm{D}[\mathrm{u}] \mathrm{M}[\mathrm{etz}]$

Historique : François $\mathrm{I}^{\mathrm{er}}$ comme l'indique l'emblématique ; au Garde-Meuble à Paris en 1542 et $1552^{25}$; au Garde-Meuble à Paris en $1666^{26}$; transformée en 1689 pour être installée à Saint-Germain-en-Laye ${ }^{27}$; incluse dans le $\mathrm{n}^{\circ} 55$ du chapitre des « Meubles de broderie sur toutes sortes de fonds » de l'inventaire de $1729^{28}$; brûlée en 1784-1785 ${ }^{29}$. Des sujets communs avec le lit $n^{\circ} 10$ ci-dessous (?).

14 3. Une autre tenture de tapisserie de broderie d'or et soye composée de huit pièces, dans chacune desquelles il y a neuf octogones, dans quatre desquels sont représentées les armes de Navarre, dans les quatre autres des compartimens de retaille et dans celuy du milieu une histoire de l'Ancien Testament, dans une bordure fonds de satin noir avec des grands escriteaux, contenant 22 aunes de cours sur 2 aunes 3/4 de hault. [Annotation en marge :] Deschargé idem et porté au no $1384 . \mathrm{D}[\mathrm{u}] \mathrm{M}[\mathrm{etz}]$

Historique: peut-être la tenture anticatholique brodée par Jeanne d'Albret, connue sous le nom de «tenture des Prisons brisées ou Prisons rompues ${ }^{30}$ »; sans doute 
l'ensemble transporté de Pau à Nérac en $1578^{31}$, puis parvenu à Fontainebleau en $1602^{32}$ ; tendue dans la chambre du légat pontifical à Saint-Germain-en-Laye, puis retirée avant son arrivée ${ }^{33}$; employée à Reims en 1610 pour le sacre de Louis XIII ${ }^{34}$; la même (?) avec son dais à Pau en $1634^{35}$; passée chez Michel Particelli d'Émery, surintendant des finances, puis en partie détruite par sa veuve en $1650^{36}$; la même (?), au Garde-Meuble à Paris en $1666^{37}$; employée au Palais-Royal en 1666 et en 1667, à Versailles en $1668^{38}$; transformée en 1689 pour être installée à Saint-Germain-en-Laye ${ }^{39} ; n^{\circ} 56$ du chapitre des « Meubles de broderie sur toutes sortes de fonds » de l'inventaire de $1729^{40} ;$ brûlée en $1785^{41}$. Le dais correspondant peut-être au $\mathrm{n}^{\circ} 7$ des dais.

16 4. Une autre tenture de tapisserie de velours découppé rouge cramoisy, fonds de satin par compartimens avec des carrez de petit point représentant des emblesmes et des fleurons, appellé la tapisserie de la reyne Claude, composée de six pièces au milieu desquelles il y a un grand rond de petits points dans un carré sur lequel est représenté une salemandre au pied d'un laurier entre deux escussons avec ces mots Extinguo nutrisco, contenant 21 aune de cours sur trois aunes $2 / 3$ de hault doublée de toille bleue. [Annotation en marge :] Deschargé idem et porté aux n 1381 et 1382. D[u] M[etz]

Historique : François $\mathrm{I}^{\mathrm{er}}$; au Garde-Meuble à Paris en 1542 et $1552^{42}$; transformée en 1689 pour être installée à Saint-Germain-en-Laye ${ }^{43} ; n^{0} 53$ et 54 du chapitre des «Meubles de broderie sur toutes sortes de fonds » de l'inventaire de $1729^{44}$; brûlée en $1785^{45}$; le dais correspondant au $\mathrm{n}^{\circ} 2$ du chapitre des dais (?).

18 5. Une autre tenture de tapisserie fort vieille de broderie d'or et soye représentant les Neuf Preux rapportée sur un fonds de velours rouge cramoisy avec des lames d'or, contenant 20 aunes de cours sur 2 aunes 11/12 de hault en huit pièces, la neufiesme ne s'estant point trouvée. [Annotation en marge :] Deschargé idem et porté au $\mathrm{n}^{\circ} 1385$. $\mathrm{D}[\mathrm{u}] \mathrm{M}[\mathrm{etz}]$

19 Historique : les rois de Navarre ; mentionnée à Pau dès $1533^{46}$; passée de Pau à Nérac en $1578^{47}$; parvenue en 1602 à Fontainebleau ${ }^{48}$; employée à Reims en 1610 pour le sacre de Louis XIII ${ }^{49}$; au Garde-Meuble à Paris en $1666^{50}$; transformée en 1689 pour être installée à Saint-Germain-en-Laye ${ }^{51} ; \mathrm{n}^{0} 57$ du chapitre des "Meubles de broderie sur toutes sortes de fonds » de l'inventaire de $1729^{52}$; mise en vente en $1752^{53}$; brûlée en $1785^{54}$.

6. Une tenture de tapisserie en broderie d'or et de soye à grands personnages rapportez sur un fonds de velours vert représentant diverses histoires de l'Ancien Testament en unze pièces, au hault desquelles sont les armes de Bourbon avec des escriteaux et par le bas des chiffres soustenus par un lion d'un costé et un griffon de l'autre, contenant vingt-quatre aunes $3 / 4$ de cours sur 2 aunes $7 / 8$ de hault. [Annotation en marge:] Deschargé partie suivant le certifficat du $30^{\mathrm{e}}$ mars $1700 . \mathrm{D}[\mathrm{u}] \mathrm{M}[\mathrm{etz}]$. Voyez le journal du garde-meuble fol. $131 \mathrm{v}^{\circ}$.

21 Historique : les Bourbons; les rois de Navarre ; sans doute la tenture passée de Pau à Nérac en $1578^{55}$ et parvenue en 1602 à Fontainebleau ${ }^{56}$; employée à Reims en 1610 pour le sacre de Louis XIII ${ }^{57}$; au Garde-Meuble à Paris en $1666^{58}$; transformée en 1689 pour être installée à Saint-Germain-en-Laye ${ }^{59} ; \mathrm{n}^{0} 2$ du chapitre des « Meubles de broderie sur toutes sortes de fonds » de l'inventaire de 1729 ; inscrite également, après modification, au $\mathrm{n}^{\circ} 58$ du même chapitre ${ }^{60}$; mise en vente en $1752^{61}$; brûlée en $1785^{62}$. Sans doute assortie au dais $\mathrm{n}^{\circ} 6$.

22 7. Une tenture de tapisserie représentant un rocher au milieu d'une mer agitée de tempeste, auquel est attaché un ancre porté par une main qui sort des nues sur un 
fonds de velours noir, dans une bordure aussy de velours noir avec des escriteaux tout autour et aux quatre coins les armes de Navarre, contenant 20 aunes de cours sur 3 aunes de hault en huit pièces fort vieilles et hors d'estat de servir.

Historique: brodée par Jeanne d'Albret; à Pau dès $1578^{63}$; parvenue en 1602 à Fontainebleau ${ }^{64}$; au Garde-Meuble à Paris en $1666^{65} ; n^{0} 3$ du chapitre des « Meubles de broderie sur toutes sortes de fonds » de l'inventaire de $1729^{66}$. Dais correspondant sous le $n^{\circ} 9$.

8. Un emmeublement de satin rouge cramoisy en broderie d'or, où il y a des ovalles d'histoires de l'Ancien Testament, garny de crespine, frange et mollet d'or, doublé de taffetas rouge et cramoisy, consistant en un lit complet, deux fauteuils, cinq sièges plians, un tapis de table et un daiz, ledit lit composé de trois pantes, quatre cantonnières et trois sousbassemens de satin en broderie d'or avec ovalles d'histoires et de trois rideaux, le fonds, dossier, trois pantes de dedans, quatre fourreaux de pilliers et la courtepointe de satin rouge cramoisy chamarrez de galon d'or ; quatre pommes de satin brodé avec leurs glands ; trois matelas de futaine et un chevet; le bois du lit de six pieds de large sur sept pieds $1 / 2$ de hault; les deux fauteuils de satin en broderie sur leurs bois ; cinq sièges plians de satin garnis d'un gallon d'or avec leurs bois; le tapis de table de satin en broderie avec son bois; le daix composé de trois pantes de dehors en broderie et de trois pantes de dedans fonds et queue de satin chamarré de galon d'or.

Historique : inconnu; semble dater du XvI ${ }^{\mathrm{e}}$ siècle; $\mathrm{n}^{\circ} 4$ du chapitre des «Meubles de broderie sur toutes sortes de fonds» de l'inventaire de 1729 (qualifié d'« ancien ») ${ }^{67}$; mis en vente en $1751^{68}$. Le dais correspondant au $n^{\circ} 15$. Un rapprochement est envisageable avec la broderie illustrant L'Adoration du Veau d'or conservée au musée de la Renaissance à Écouen (inv. E. Cl. 1488), comme nous le suggère Muriel Barbier (fig. 1) 
Figure 1

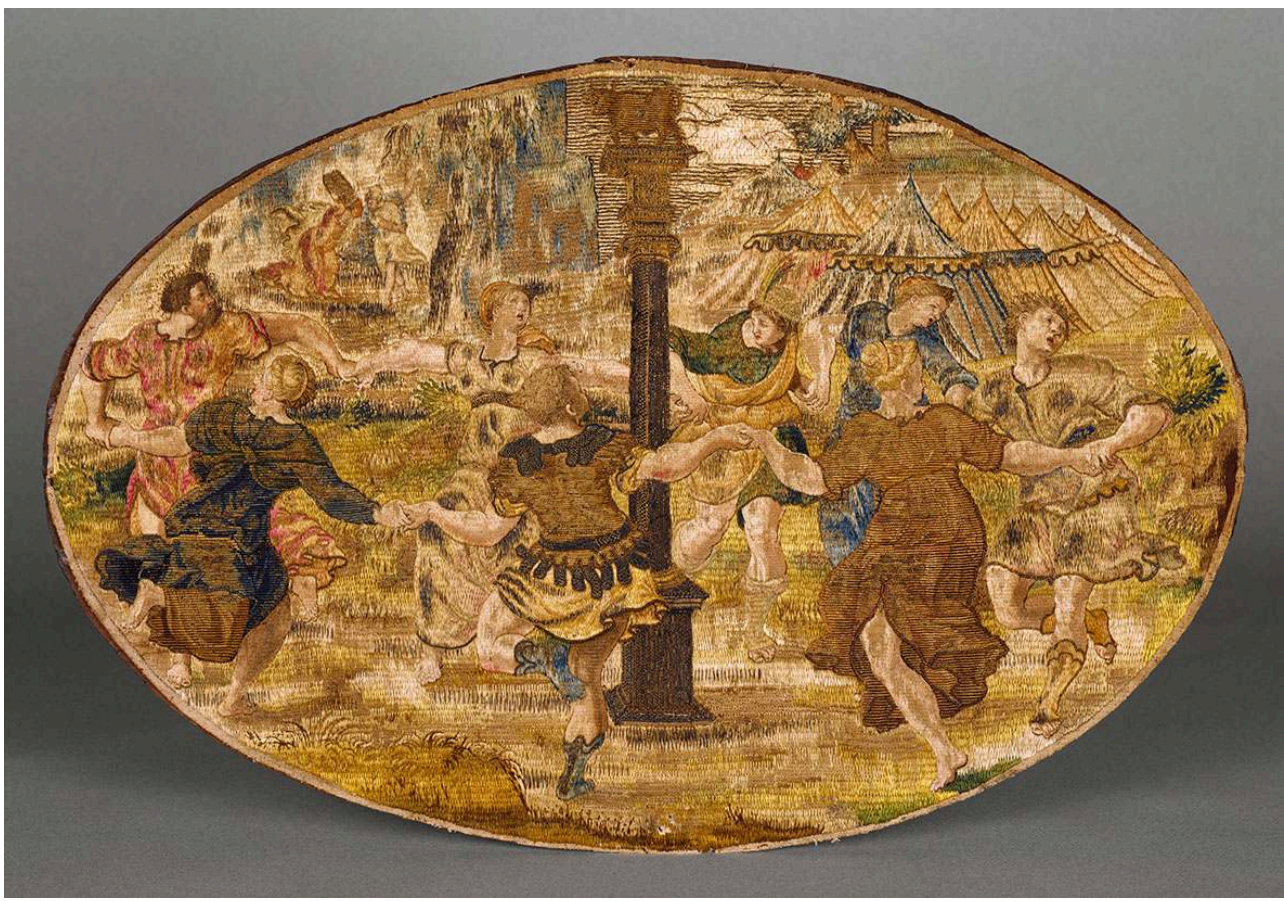

L'Adoration du Veau d'or, broderie, soie et métal précieux sur lin, XVle siècle, 34 × $50 \mathrm{~cm}$. Écouen, musée national de la Renaissance (inv. E. Cl. 1488).

Phot. Gérard Blot. @ RMN-Grand Palais (musée de la Renaissance, château d'Ecouen).

9. Un lit my-party [c'est-à-dire associant deux tissus différents] de velours violet en broderie et de drap d'or frisé très riche composé de dix-huit pièces, sçavoir trois pantes de dehors, trois pantes de dedans, 2 sousbassemens, le fonds et le dossier my-party de velours violet en broderie et taillure de toille d'or et riche brocat d'or frisé comme dessus, appellé anciennement le lit d'Angleterre à cause des armes d'Angleterre qui sont au milieu du dossier avec la devise Honni soit qui mal y pense, et de la courtepointe de taffetas violet picqué avec un cordonnet d'or, trois rideaux et quatre cantonnières aussy de taffetas violet chamarrez d'un guillochis d'or; un petit rond de mesme velours, brocat et broderie, au milieu duquel sont les armes d'Angleterre. Nota qu'il y a trois cantonnières et un rideau de fort meschant taffetas et qu'il manque du molet à un costé du dossier et la frange au bas d'une bonne-grâce et que la frange des pantes est coupée en quelques endroits.

Historique : sans doute Henry VIII d'Angleterre ; sans doute donné à François $\mathrm{I}^{\mathrm{er}}$ en 1532 lors de l'entrevue de Boulogne ${ }^{69} ; \mathrm{n}^{\circ} 5$ du chapitre des « Meubles de broderie sur toutes sortes de fonds » de l'inventaire de 1729 (qualifié de « vieux ») ${ }^{70}$; détruit en $1745^{71}$.

10. Un lit imparfait [c'est-à-dire inachevé] de velours canelé cramoisy remply de plusieurs figures de Métamorphoses en broderie de relief or, argent et soye, appellé anciennement le Cerf fragile, consistant en deux sousbassemens, le fonds où sont représentées des harpies avec quatre termes et le dossier, six pantes d'une autre manière de dessein et broderie et d'une couverture de parade qui n'a nul rapport aux autres pièces estant de velours rouge cramoisy et fueuillages de toille d'or où il y a une salemandre dans le milieu, le tout sans frange, mollet ny doublure. Nota que la dite couverture de parade est fort en désordre. [Annotation en marge :] Deschargé suivant 
le certifficat du $30^{\mathrm{e}}$ mars 1700 à l'exception du dossier. $\mathrm{D}[\mathrm{u}] \mathrm{M}[\mathrm{etz}]$. Voyez le journal fol. 132 .

Historique : François I ${ }^{\mathrm{er}}$; au Garde-Meuble à Paris en 1542 et $1552^{72}$; transformé en 1689 pour être installé à Saint-Germain-en-Laye ${ }^{73}$; inclus dans le $n^{\circ} 52$ du chapitre des «Meubles de broderie sur toutes sortes de fonds » de l'inventaire de $1729^{74}$; brûlé en $1784^{75}$. Pourrait accompagner la tenture des Métamorphoses $\mathrm{n}^{0} 2$ ci-dessus.

11. Un lit imparfait tout de broderie à personnages représentant l'histoire de Joseph, contenant trois pantes, 2 sousbassemens, le fonds et le dossier. Nota que le tout est sans doublure, frange ny mollet et assez beau et riche excepté le fonds qui est gasté. [Annotation en marge :] Deschargé idem à l'exception du fonds. D[u] M[etz]. Voyez le journal idem.

Historique : inconnu, paraît ancien; transformé en 1689 pour être installé à SaintGermain-en-Laye ${ }^{76}$; inclus dans le $\mathrm{n}^{\circ} 52$ du chapitre des "Meubles de broderie sur toutes sortes de fonds » de l'inventaire de $1729^{77}$; brûlé en $1784-1786^{78}$.

12. Un autre lit imparfait de velours tanné cramoisy tout remply de figures de broderie d'or et d'argent embouties représentant l'Enlèvement de la belle Hélène, consistant en trois pantes, deux sousbassemens, fonds et dossier. Nota que le tout est assez beau quoyqu'usé sans doublure, frange ny molet. [Annotation en marge :] Deschargé idem à l'exception du fonds et dossier. Voyez le journal fol. $132 \mathrm{v}^{\circ}$. D[u] M[etz].

Historique : inconnu, paraît ancien; transformé en 1689 pour être installé à SaintGermain-en-Laye (cite « l'incendie de Troyes ») ${ }^{79}$; brûlé en $1784^{80}$.

[...]

33. Un lit imparfait de point et de broderie de soye platte, appellé anciennement le lit des Satyres, consistant en un fonds, un dossier et six pantes, le fonds de satin de Bruges, le dossier où est représenté une Diane et des vers aux deux costez, quatre des pantes remplies de figures de femmes et de satyres avec des escriteaux et les deux autres toutte d'escriture sur satin de Bruges blanc garnies de grande frange d'or supportée de soye verte.

Historique : brodé par Jeanne d'Albret ; parvenu en 1602 à Fontainebleau ${ }^{81} ; n^{\circ} 6$ du chapitre des « Meubles de broderie sur toutes sortes de fonds » de l'inventaire de 1729 $(« \text { fort vieux } »)^{82}$.

38. Un pavillon de damas violet à petittes fleurs appellé le pavillon des Sacres, aux deux costez duquel il y a une bande de velours violet parsemée de fleurs de lis d'or gauffré, ledit pavillon garny de mollet tout autour avec son chapiteau de velours violet aussy parsemé de fleurs de lis d'or gauffré garny de frange et molet d'or.

Historique : inconnu; $\mathrm{n}^{\circ} 7 \mathrm{du}$ chapitre des $«$ Meubles de broderie sur toutes sortes de fonds » de l'inventaire de $1729^{83}$.

39. Un lit en désordre [c'est-à-dire présentant des incohérences] de velours rouge cramoisy brun semé de fueuillages de houx de broderie d'or avec figures de broderie relevée et amboutie et escussons des armes de Bourbon, garny de crespine, frange et mollet d'or faux, composé de six pantes, 4 cantonnières, fonds, dossier, trois sousbassemens, courtepointe et deux fourreaux de pilliers de velours rouge brun, garny partout de broderie comme dessus et de trois rideaux de brocat or et argent et violet doublez de taffetas rouge, appellé anciennement le lit de Melluzine à cause qu'elle est dépeinte dans une fontaine de broderie sur le dossier ; quatre pommes et quatre glands 
faux ; trois matelas de futaine; un bois de lit de six pieds de large sur sept pieds $1 / 2$ de hault; un tapis de table dudit velours et broderie. [Annotations en marge :] Veu le certifficat du $30^{\circ}$ mars 1700 , fol. 3 , deschargé 4 cantonnières. $\mathrm{D}[\mathrm{u}] \mathrm{M}[\mathrm{etz}]$. Deschargé les matelas et le bois de lict suivant le certifficat du $30^{\circ}$ mars $1700 . \mathrm{D}[\mathrm{u}] \mathrm{M}[\mathrm{etz}]$.

Historique : les Bourbons (Charles de Bourbon, duc de Vendôme (1489-1537) (?)) ; les rois de Navarre ; passé de Pau à Nérac en $1578^{84}$; parvenu à Fontainebleau en $1602^{85}$; transformé en 1689 pour être installé à Saint-Germain-en-Laye ${ }^{86} ; n^{\circ} 8$ du chapitre des «Meubles de broderie sur toutes sortes de fonds » de l'inventaire de 1729 (cite en outre des «moutons emparqués » ${ }^{87}$; détruit en $1745^{88}$; le dais correspondant au $n^{\circ} 8 \mathrm{du}$ chapitre des dais.

40. Un riche emmeublement tout de broderie fonds d'or à grains d'orge avec cartouches et camoyeux relevez d'or, représentant l'histoire de Moyse, garny de campanne, consistant en un lit complet, quatre fauteuils, dix-huit sièges plians, un tapis de table, un escran et le daix semblables, ledit lit composé de six pantes, 4 cantonnières, le fonds et le dossier de riche broderie relevée de trois sousbassemens, d'une campanne, de la courtepointe, trois rideaux et 2 fourreaux de pilliers de broderie moins relevée avec plusieurs emblesmes; les quatre pommes et leurs plumes; les quatre housses de fauteuils et dix-huit sièges de broderie moins relevée avec des emblesmes attachez sur leurs bois ; le tapis de table et l'escran de la mesme broderie; le daiz composé de sept pantes et le fonds de riche broderie avec cartouches et camayeux et la queue d'une pièce de tapisserie séparée ; trois matelas de satin blanc et un de futaine et un chevet; le bois du lit de six pieds $1 / 2$ sur huit de hault.

Historique : généralement daté du $\mathrm{XVI}^{\mathrm{e}}$ siècle, mais plus vraisemblablement exécuté pour le cardinal de Richelieu ; figure à son inventaire après décès qui cite les broderies des « histoires de l'Exode » et lui accorde l'importante prisée de 45000 livres $^{89}$; légué au roi ${ }^{90}$; employé en 1660 à Saint-Jean-de-Luz pour le mariage de Louis XIV ${ }^{91}$; le même (?) employé en 1722 pour le sacre de Louis XV ${ }^{92} ; \mathrm{n}^{\circ} 9$ du chapitre des « Meubles de broderie sur toutes sortes de fonds " de l'inventaire de 1729, qui le qualifie de «meuble du Sacre » et le décrit longuement; le texte mentionne sur le fond du dais « la Création de l'homme", sur les pentes des "tableaux ovalles représentant différens sujets de l'Histoire Sainte », sur la queue « dans sept cartouches, des sujets de l'histoire de Moyse ", sur la bordure "sept tableaux représentant les vertus théologalles et cardinalles", "des attributs et instrumens d'artillerie par le bas et des trophées d'armes ", ainsi que l'« écusson du cardinal de Richelieu par en-haut " ${ }^{93}$; le même (?) employé en 1775 pour le sacre de Louis XVI ${ }^{94}$; brûlé en 1784 et $1786^{95}$; dais correspondant au $\mathrm{n}^{\circ} 19$. Le médaillon brodé avec L'Adoration du Veau d'or, conservé au musée de la Renaissance à Écouen (inv. E. Cl. 1488), a été mis en relation, apparemment à tort, avec cet ensemble ${ }^{96}$.

43. Un autre daiz plus ancien de velours violet garny de frange d'or ; un fauteuil et six carreaux pour accompagner ledit daiz.

Historique : Henri IV ; $\mathrm{n}^{\circ} 11$ du chapitre des « Meubles de broderie sur toutes sortes de fonds » de l'inventaire de 1729 (qui cite notamment « six chiffres de Henry quatre et six de Marie de Médicis $")^{97}$; inventorié également au $\mathrm{n}^{0} 22$ du chapitre des dais.

[...]

88. Un emmeublement de velours tanné rosin cramoisy en broderie avec camoyeux d'histoires de l'Ancien Testament doublé de riche brocat d'or et d'argent fonds rouge, le 
tout garny de campanne et mollet de broderie d'or et d'argent fonds rouge, le tout garny de campanne et mollet de broderie d'or, d'argent et soye, consistant en un lit complet, 6 fauteuils, 6 chaises, six sièges plians et un tapis de table, ledit lit de 6 pieds $\frac{1}{2}$ de large sur $8 \frac{1}{2}$ de hault composé de trois pantes, 4 bonnes-grâces, 3 sousbassemens, quatre rideaux de velours en broderie par bandes, le fonds, dossier, petittes pantes et courtepointe dudit brocat, quatre pommes de velours en broderie, les 6 fauteuils, six chaises et 6 sièges plians dudit velours en broderie garnys de campannes, les bois desdits fauteuils, chaises et sièges et le bois du lit.

Historique : brodé par Rémy Dubois pour Anne d'Autriche en 1638 et employé pour la naissance du futur Louis XIV ; le cardinal Mazarin dès $1657^{98}$; acquis par Louis XIV en $1665^{99}$; brûlé en $1784^{100}$. Bien que datant du XVII ${ }^{\mathrm{e}}$ siècle, le lit est cité ici en raison de ses liens avec le $\mathrm{n}^{\circ}$ suivant.

89. Une tenture de tapisserie de velours tanné rosin cramoisy à crotesques, dessein de Raphaël, en broderie d'or, d'argent et soye rapportée sur ledit velours, composée de neuf pièces, dans le milieu de chacune desquelles il y a une grande ovalle aussy en broderie, où sont représentez les divertissemens de François premier et de Henry second, ladite tapisserie de 23 aunes $3 / 4$ de cours sur 3 aunes moins $1 / 16$ de hault doublée de toille rouge.

Historique : brodée par Robert Mestays pour Diane de Poitiers (1500-1566) en 1553 ${ }^{101}$; Gabrielle d'Estrées ${ }^{102}$; le cardinal Mazarin dès $1653^{103}$; acquise par Louis XIV en $1665^{104}$; $\mathrm{n}^{\circ} 22 \mathrm{du}$ chapitre des «Meubles de broderie sur toutes sortes de fonds » de l'inventaire de 1729 , qui décrit longuement l'ensemble constitué des $n^{\circ} 88$ et $89^{105}$; brûlée en $1784^{106}$. Subsistent deux médaillons brodés au musée des Tissus de Lyon $^{107}$ (fig. 2, fig. 3). 
Figure 2

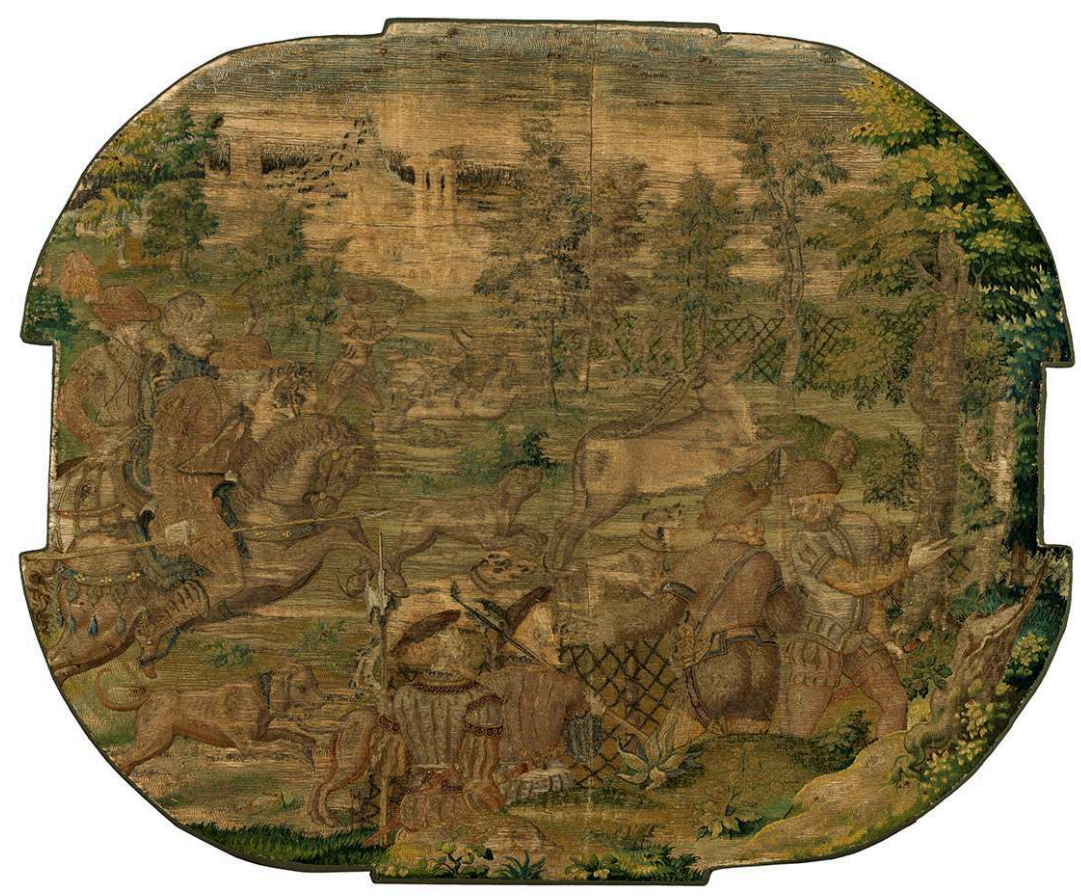

Robert Mestays, La Chasse au cerf, broderie, soie, métal précieux et peinture, 1553, 84,6 x 103,3 cm, Lyon, musée des Tissus (inv. MT 25438).

Phot. Pierre Verrier. (C) Musée des Tissus et des Arts décoratifs, Lyon. 


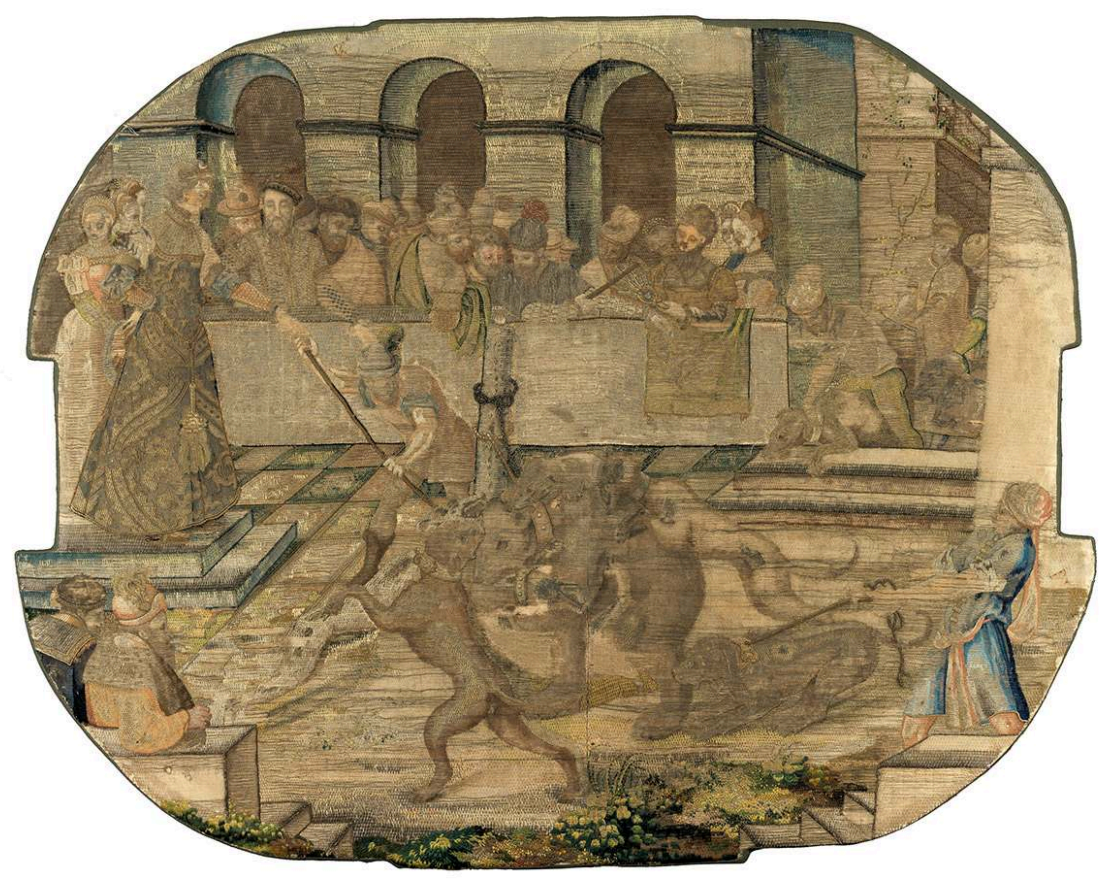

Robert Mestays, Le Combat de l'ours, broderie, soie, métal précieux et peinture, 1553, 82,1 × 104,2 cm, Lyon, musée des Tissus (inv. MT 25495).

Phot. Pierre Verrier. (C) Musée des Tissus et des Arts décoratifs, Lyon.

\section{Daiz $^{108}$}

1. Un daiz d'un entrelas de velours et satin rouge en broderie par bandes en ronds sur un fonds de velours vert aussy en ronds, le tout rapporté sur un fonds de velours blanc à ramages liséré d'un cordonnet d'or, ledit daiz composé de son fonds, queue et pantes garnies d'une grande frange de soye rouge cramoisy. Nota que led. daix est fort vieux et qu'il y manque 61 rosettes de drap d'or.

Historique: les rois de Navarre/Henri IV (?); $\mathrm{n}^{\circ} 1$ du chapitre des «Dais» de l'inventaire de $1729^{109}$; mis en vente en $1741^{110}$ et adjugé 377 livres à la dame Lépine ${ }^{111}$.

2. Un autre daiz de velours rouge cramoisy, fonds de satin par compartimens avec des carrez de petits points représentant des devises, appellé le daiz de la reyne Claude, composé de fonds, queue et trois pantes doubles garnies d'une crespine d'or et d'argent par coupons avec une souspante de soye; au milieu du fonds il y a un rond de petit point dans lequel est représenté une salemandre au pied d'un laurier avec ces mots Extingo nutrisco. Deschargé suivant le certifficat du $30^{\mathrm{e}}$ mars 1700. D[u] M[etz].

Historique : François I ${ }^{\mathrm{er}}$; transformé en 1689 pour être installé à Saint-Germain-en-Laye (des «tableaux octogonnes de petit point » sont cités) ${ }^{112}$; déchargé de l'inventaire de $1729^{113}$; les restes mis en vente en $1751^{114}$. À associer peut-être à la tenture ${ }^{\circ} 4$.

54 3. Un daiz fait au petit point composé de son fonds, queue et pantes; au fonds il y a des soleils et des escriteaux dans de grands carrez fonds bleu et à la queue, dans le milieu, 
deux sphères et des devises aux costez; ses trois pantes doublées de taffetas blanc garnies de frange d'or supportées de frange de soye noire le tout doublé de toille rouge.

Historique : les rois de Navarre ; semble-t-il à Pau dès $1533^{115}$; passé de Pau à Nérac en $1578^{116}$; à Nérac en $1598^{117}$; à Fontainebleau en $1602^{118} ; n^{0} 3$ du chapitre des « Dais » de l'inventaire de $1729^{119}$; mis en vente en $1741^{120}$ et adjugé 540 livres à Lefranc, marchand rue Saint-Honoré ${ }^{121}$.

4. Un daiz de velours vert parsemé de flames d'or et lames d'argent en broderie sur le fonds duquel et sur la queue il y a une grande salemandre aussy en broderie fort emboutie, les pantes garnies de frange d'or et soye verte par coupons le tout doublé de toille bleue.

Historique : François I ${ }^{\mathrm{er}} ; \mathrm{n}^{\circ} 4$ du chapitre des « Dais » de l'inventaire de 1729 («apellé le dais de la Salemandre $»)^{122}$; mis en vente en $1752^{123}$.

5. Un daiz de tapisserie de haulte lisse d'or et soye appellé le daiz de la Charité, composé du fonds, de la queue et de deux pantes doubles garnies de crespine par boutons d'or, d'argent et soye ; au milieu du fonds est représenté un pélican et huit anges à l'entour et sur la queue une grande femme qui porte un enfant et donne à téter à un vieillard.

Historique: François I ${ }^{\text {er }}$; employé en 1532 lors de l'entrevue de Boulogne avec Henry VIII" ${ }^{124}$; au Garde-Meuble à Paris en 1542 et $1552^{125} ; \mathrm{n}^{\circ} 5$ du chapitre des « Dais » de l'inventaire de $1729^{126}$; brûlé en 1784 et $1787^{127}$.

6. Un daiz de velours vert sans queue, dont le fonds est brodé de l'histoire de Judich, figures presque de grandeur naturelle dans un grand rond, les trois pantes doublées de satin vert garnies de frange d'or faux supportées d'une frange de soye verte. Deschargé suivant le certifficat du $30^{\mathrm{e}}$ mars 1700 . Voyez le journal fol. $133 \mathrm{v}^{\circ}$. D [u] M[etz].

61 Historique: les rois de Navarre; passe de Pau à Nérac en $1578^{128}$; parvenu à Fontainebleau en $1602^{129}$; transformé en 1689 pour être installé à Saint-Germain-enLaye ${ }^{130}$; déchargé de l'inventaire de $1729^{131}$; les restes mis en vente en $1752^{132}$. À associer sans doute à la tenture $\mathrm{n}^{\circ} 6$.

7. Un daiz fort en désordre d'un compartiment de satin rouge cramoisy brodé d'un entrelas de paillettes d'or par carrez fonds de velours vert avec un chiffre et octogones, composé du fonds, queue et trois pantes, ayant au milieu du fonds un soleil et au milieu de la queue un tableau de petits points qui représentent un homme et une femme qui dit « Da mihi liberos ", le tout sans frange, crespine ny mollet.

Historique : Sans doute brodé par Jeanne d'Albret; peut-être cité à Nérac en $1552^{133}$; peut-être à Nérac en $1569^{134}$, puis passé de Nérac à Pau en $1570^{135}$; peut-être passé de Pau à Nérac en $1578^{136}$, puis parvenu à Fontainebleau en $1602^{137} ; n^{\circ} 7$ du chapitre des «Dais » de l'inventaire de $1729^{138}$; mis en vente en $1741^{139}$ et adjugé 550 livres à Languilleux ${ }^{140}$. À associer peut-être aux meubles $n^{\circ} 1$ et $n^{\circ} 3$.

64 8. Un daiz de velours rouge cramoisy en broderie, brodé de grands personnages fort embouties et de fueuillages de houx d'or et d'argent assortissant au lit appellé de Mellusine, composé de son fonds, queue et trois pantes doubles garnies de crespine d'or supportées d'une grande frange de soye rouge cramoisy doublé de toille rouge.

65 Historique : voir le lit $\mathrm{n}^{\circ} 39 ; \mathrm{n}^{\circ} 8$ du chapitre des «Dais» de l'inventaire de $1729^{141}$; détruit en $1746^{142}$. 

$1602^{161}$; transformé en 1689 pour être installé à Saint-Germain-en-Laye ${ }^{162}$; passé du chapitre des « Dais » à celui des « Meubles de broderie sur toutes sortes de fonds » sur l'inventaire de $1729^{163}$; les restes mis en vente en $1751^{164}$; brûlé en $1784^{165}$. d'argent en satin rouge cramoisy liseré d'un cordonnet d'or, composé du fonds, queue et trois pantes garnies de frange d'or et soye doublées de damas moittié vert et moittié blanc, le tout doublé de toille rouge.

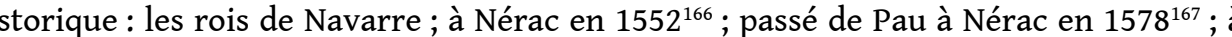
Fontainebleau en $1602^{168} ; n^{\circ} 13$ du chapitre des « Dais » de l'inventaire de $1729^{169}$; mis en vente en $1741^{170}$ et adjugé 900 livres à Berthe ${ }^{171}$. inventorié au chapitre des meubles $\mathrm{n}^{\circ} 1^{\mathrm{er}}$.

Historique : voir le meuble $\mathrm{n}^{\circ} 1$; hypothétiquement cité à Nérac $1569^{172}$, avec une confusion possible avec le dais $\mathrm{n}^{\circ} 7 ; \mathrm{n}^{\circ} 14$ du chapitre des «Dais» de l'inventaire de 1729 ( $\left(\right.$ avec chiffres AL en or », sans doute pour AJ) ${ }^{173}$. 
79 15. Un daiz de satin rouge cramoisy en broderie d'or, inventorié au chapitre des meubles $\mathrm{n}^{\circ} 8$. chapitre des «Dais » de l'inventaire de 1729 (longuement décrit; le document cite les chiffres d'Henri IV et de Marie de Médicis et y associe un tapis de pied) ${ }^{183}$; brûlé en $1785^{184}$.

Historique : voir le meuble $\mathrm{n}^{\circ} 8 ; \mathrm{n}^{\circ} 15$ du chapitre des « Dais » de l'inventaire de 1729 (plus longuement décrit) ${ }^{174}$.

16. Un daiz de velours rouge cramoisy enrichy de taillure de brocat d'or frisé et de chiffre en broderie d'argent à grains d'orge, composé de son fonds, queues et trois pantes doublées de taffetas rouge cramoisy garnies de frange d'or supportées de soye rouge cramoisy, le tout doublé de toille rouge.

Historique: inconnu; $\mathrm{n}^{\circ} 16 \mathrm{du}$ chapitre des «Dais» de l'inventaire de 1729 $(« \text { vieux } »)^{175}$.

17. Un daiz de serge de soye bleue mourant parsemé de lions de taffetas jaune liseré de soye rouge qui porte un escusson sans frange, crespine ny mollet doublé de toille rouge.

Historique : inconnu ; $\mathrm{n}^{\circ} 17$ du chapitre des « Dais » de l'inventaire de 1729 (« vieux ») (176 $^{\circ}$ ; mis en vente en $1751^{177}$.

18. Un daiz de satin bleu en broderie de toille d'or et de velours rouge cramoisy découpé par compartimens différens, composé de fonds, queue et trois pantes doublées de satin bleu garnies de frange de soye fueuille morte et bleu par coupons, le tout doublé de toille bleue.

Historique : inconnu ; $\mathrm{n}^{\circ} 18$ du chapitre des « Dais » de l'inventaire de 1729 (« vieux ») (178 $^{178}$ ; mis en vente en $1741^{179}$ et adjugé 425 livres à Bricogne ${ }^{180}$.

19. Un daiz de l'emmeublement de l'histoire de Moyse enregistré au chapitre des lits et emmeublemens $\mathrm{n}^{\circ} 40$.

Historique : voir le meuble $\mathrm{n}^{\circ} 40 ; \mathrm{n}^{\circ} 19$ du chapitre des « Dais » de l'inventaire de 1729 (longuement décrit, exactement comme au $n^{\circ} 9$ du chapitre des « Meubles de broderie ... »; le document cite notamment « l'écusson du cardinal de Richelieu ») ${ }^{181}$; brûlé en $1784^{182}$.

$[\ldots]$

22. Un autre daiz aussy de velours violet parsemé de fleurs de lis d'or enregistré audit chapitre $\mathrm{n}^{\circ} 43$.

23. Un daiz de toille d'or à fleurs de velours vert avec bandes de broderie, composé du fonds, queue et trois pantes garnies de frange et mollet d'or et d'argent.

Historique : Henri II ; $n^{\circ} 23$ du chapitre des « Dais » de l'inventaire de 1729 (le document cite des « chiffres de Henry II ») ${ }^{185}$; mis en vente en $1751^{186}$.

[...]

26. Un daiz de tapisserie de laine et soye relevée d'or entouré de bandes de velours rouge cramoisy avec des grands chiffres de drap d'or, composé du fonds dans lequel sont représentées deux bergères et un berger, de la queue qui représente le Jugement de Pâris et de trois pantes garnies de crespine d'or à la milanoise supportées d'une frange de soye rouge cramoisy et doublées de brocat d'or trait et façonné. 

en $1644^{187}$; acquis par Louis XIV en $1665^{188} ; n^{\circ} 26$ du chapitre des « Dais » de l'inventaire de 1729 («dessein de Julles Romain, fabrique de Bruxelles », "brodé des chiffres de Guise AG (?) de drap d'or couronnés ») ${ }^{189}$; brûlé en $1784^{190}$.

77. Un autre plus grand daiz de tapisserie de laine et soye relevée d'or aussy entouré de bandes de velours rouge cramoisy avec de grands chiffres de drap d'or, composé seulement du fonds où est représenté Jupiter assis sur son aigle tenant une pomme d'or accompagné de plusieurs divinitez, de nimphes et quelques faunes, et de trois pantes qui représentent l'enlèvement d'Hélène garnis de frange d'or à la milanoise suportée d'une soye et doublée de toille d'or trait.

Historique : les ducs de Guise; cité à l'inventaire de Charles de Lorraine, duc de Guise, en $1644^{191}$; acquis par Louis XIV en $1665^{192} ; \mathrm{n}^{\circ} 27$ du chapitre des « Dais » de l'inventaire de 1729 («aussi dessein de Julles Romain, fabrique de Bruxelles », "brodé aussi des chiffres de Guise de toille d'or couronnés $»)^{193}$; brûlé en $1784^{194}$.

[...]

100 33. Un autre daiz de velours bleu parsemé de fleurs de lis en broderie d'or goffré composé du fonds, queue et de trois pantes doubles garnies de crespine supportée d'un grande frange de soye bleue.

Historique : inconnu; $n^{0} 33$ du chapitre des «Dais » de l'inventaire de 1729 («vieux », " parsemé de grandes fleurs de lis, branchages et animaux d'or ») ${ }^{195}$; détruit en $1746^{196}$.

34. Un daiz de broderie d'or, d'argent et soye représentant plusieurs histoires de l'Ancien Testament, composé du fonds, queue et trois pantes doubles garnies de grande campanne en broderie à jour avec des houppes d'or et soye ; sur le fonds est représenté Moyse sur la montagne recevant les tables de la Loy dans un grand compartiment accompagné hault et bas de quatre cartouches en ovalles remplies d'histoires et entre deux des armes de France et sur la queue l'histoire de la manne dans un octogone aussy accompagné de quatre cartouches en ovalles remplies d'histoires et entre deux les armes de France.

Historique: hypothétiquement les rois de Navarre; voir le dais dit des «Tables de Moyse ", passé de Pau à Nérac en $1578^{197}$; puis à Fontainebleau en $1602^{198} ; n^{\circ} 34$ du chapitre des «Dais» de l'inventaire de $1729^{199}$; brûlé en $1784^{200}$. Plus vraisemblablement un élément complémentaire du lit $n^{\circ} 40$.

\section{BIBLIOGRAPHIE}

ALCOUFFE, Daniel. « Les ventes de Louis XV ». Dans CHARLES, Jacques (dir.). De Versailles à Paris : le destin des collections royales. Cat. exp., Paris, centre culturel du Panthéon. Paris : centre culturel du Panthéon, 1989.

ALFASSA, Paul. «L'achat par Louis XIV des tapisseries des “Chasses de l'empereur Maximilien" ». Bulletin de la Société de l'histoire de l'art français, 1918-1919. 
COSNAC, Gabriel-Jules de. Mazarin et Colbert. Paris : E. Plon-Nourrit, 1892.

DEVILLE, Jules. Dictionnaire du tapissier critique et historique de l'ameublement français depuis les temps anciens jusqu'à nos jours. Paris/Liège/Berlin : s.n., 1878-1880.

DUBUISSON-AUBENAY, François-Nicolas Baudot. Journal des guerres civiles, 1648-1652. Édition Gustave Saige. Paris : H. Champion, 1883-1885.

FAVREAU, Marc. « "Description des sujets de tapisseries" : un inventaire iconographique partiel des tapisseries de la Couronne sous le règne de Louis XIV ». Bulletin de la Société de l'histoire de l'art français, 2005.

GARASSE, François. La Doctrine curieuse des beaux esprits de ce temps, ou pretendus tels ... Paris : Sébastien Chappelet, 1623.

GODEFROY, Théodore et Denys. Le Cérémonial françois... Paris : Sébastien et Gabriel Cramoisy, 1649. GUIFFREY, Jules. Histoire générale de la tapisserie. T. 1, France, Pays-Bas. Paris : Société anonyme de publications périodiques, 1878-1885.

GUIFFREY, Jules. Inventaire général du mobilier de la Couronne sous Louis XIV (1663-1715). Paris : J. Rouam, 1885-1886.

GUIFFREY, Jules. «Inventaires des meubles précieux de l'hôtel de Guise en 1644 et en 1688 et de l'hôtel de Soubise en 1787 ». Nouvelles Archives de l'art français, 1896.

HAMY, Alfred. Entrevue de François Premier avec Henry VIII, à Boulogne-sur-Mer, en 1532. Intervention de la France dans l'affaire du divorce, d'après un grand nombre de documents inédits. Paris : L. Gougy, 1898.

LABORDE, Léon de. La Renaissance des arts à la cour de France. Études sur le seizième siècle. Paris : L. Potier, 1850.

LAPRADE, Jacques de. «Au temps de Marguerite d'Angoulême, un inventaire mobilier de Nérac (1552) ». Le Vieux Papier, XXIII, 1961-1963.

LAPRADE, Jacques de. " Un inventaire des tentures et des meubles transportés de Pau à Nérac en 1578 ». Bibliothèque d'humanisme et Renaissance. Travaux et documents, XXIV, 1962.

LAPRADE, Jacques de. «Richesses anciennes du château de Pau. L'envoi à Paris de la tenture et du lit des Devises en 1597 et 1601 ». Bulletin de la Société des sciences, lettres et arts de Pau, XXV, 1964 (tiré à part non paginé).

LAPRADE, Jacques de, et PÉROT, Jacques. « La destinée du meuble de Pau sous Henri IV : les pièces envoyées à Fontainebleau en 1602 ». Provinces et pays du Midi au temps d'Henri de Navarre (1555-1589). Actes du colloque de Bayonne, 7-9 octobre 1988. Pau : château de Pau/Association Henri IV, 1989.

LEVI, Honor. « L'inventaire après décès du cardinal de Richelieu ». Archives de l'art français, XXVII, 1985.

RAHLENBECK, Charles. «Les tapisseries des rois de Navarre ». Messager des sciences et des arts de la Belgique, 1868.

RAYMOND, Paul. « Notes extraites des comptes de Jeanne d'Albret et de ses enfants, 1556-1608 ». Revue d'Aquitaine et des Pyrénées, X, 1866, XI, 1867.

SCHNEEBALG-PERELMAN, Sophie. «Richesses du garde-meuble parisien de François I ${ }^{\mathrm{er}}$. Inventaires inédits de 1542 et 1551 ». Gazette des beaux-arts, 1971/2. 
SULLY, Maximilien de Béthune, duc de. Mémoires des sages et royales œeconomies d'Estat, domestiques, politiques et militaires de Henry le Grand... Amsterdam : [1640].

THOMAS, Danièle. Inventaires mobiliers et pièces annexes : château de Pau, château de Nérac (XVI et XVII siècles). Pau : Société des amis du château, 1996.

VÉRON-DENISE, Danièle. « Un brodeur de Henri II : Robert Mestays ». Dans OURSEL, Hervé, et FRITSCH, Julia (dir.). Henri II et les Arts. Actes du colloque international organisé par l'École du Louvre et le musée national de la Renaissance-Écouen, 25, 26 et 27 septembre 1997. Paris : École du Louvre, 2003.

VÉRON-DENISE, Danièle. « Notes sur un lit brodé de Mazarin, dans lequel la reine Anne d'Autriche accoucha du dauphin, futur Louis XIV ». Dans CONIHOUT, Isabelle de, MICHEL, Patrick (dir.). Mazarin, les Lettres et les Arts. Saint-Rémy-en-l'Eau/Paris : Monelle Hayot/Bibliothèque Mazarine, 2006.

Expositions

CRÉPIN-LEBLOND, Thierry, THIRION, Jacques (dir.). Parures d'or et de pourpre. Le mobilier à la cour des Valois. Cat. exp., château de Blois, 15 juin-30 septembre 2002. Paris/Blois : château de Blois/ Somogy éditions d'art, 2002.

DROGUET, Vincent, CRÉPIN-LEBLOND, Thierry (dir.). Le Roi et l'Artiste. François I er et Rosso

Fiorentino. Vincent Droguet et Thierry Crépin-Leblond (dir.). Cat. exp., château de Fontainebleau, 23 mars-24 juin 2013. Paris : RMN-Grand Palais, 2013.

Henri II à Saint-Germain-en-Laye. Une cour royale à la Renaissance. Cat. exp., Saint-Germain-en-Laye, musée des Antiquités nationales, 31 mars-14 juillet 2019. Paris : RMN-GP, 2019.

\section{NOTES}

1. - Jean Vittet, est conservateur en chef du patrimoine au château de Fontainebleau, chargé du mobilier historique et des arts décoratifs avant 1815. Il a occupé des fonctions scientifiques au Mobilier national pendant vingt-quatre ans (1989-2013), institution où il était chargé du fonds des tapisseries et des modèles antérieurs à 1960. Il est l'auteur de plus d'une centaine de livres, articles et contributions à des catalogues d'exposition. Ses recherches l'ont conduit à étudier les collections royales d'objets d'art de la fin du Moyen Âge au xIx siècle ainsi que les productions des manufactures françaises de tapisserie. Il est le principal contributeur d'un ouvrage consacré à La Collection de tapisseries de Louis XIV (2010). Plus récemment (2014), il a fait paraître Les Gobelins au siècle des Lumières : un âge d'or de la manufacture royale.

2. - GUIFFREY, Jules. Inventaire général du mobilier de la Couronne sous Louis XIV (1663-1715). Paris : J. Rouam, 1885-1886, vol. II, les « Lits et emmeublemens » à partir de la p. 209, les « Daiz » à partir de la p.464. Une partie du chapitre des «Lits et emmeublemens" avait déjà été publiée, peu avant Jules Guiffrey, par Jules Deville (Dictionnaire du tapissier critique et historique de l'ameublement français depuis les temps anciens jusqu'à nos jours. Paris/Liège/Berlin : s.n., 1878-1880, p. 413-432). En général, sa transcription est plus fidèle et plus complète que celle de Guiffrey.

3. - Archives nationales, $\mathrm{O}^{1} 3330-\mathrm{O}^{1} 3333$.

4. - Comme la provenance ou la localisation Fontainebleau n'apparaît presque jamais, on peut penser que le mobilier de ce palais n'a pas été inclus dans l'inventaire, ni celui de Compiègne, qui n'est jamais cité, tandis que Saint-Germain-en-Laye, Le Val, Chambord et Marly le sont sporadiquement. 
5. - FAVREAU, Marc. " "Description des sujets de tapisseries" : un inventaire iconographique partiel des tapisseries de la Couronne sous le règne de Louis XIV ». Bulletin de la Société de l'histoire de l'art français, 2005, p. 47-67.

6. - AN, $\mathrm{O}^{1} 3334-\mathrm{O}^{1} 3341$.

7. - Selon Guiffrey, le domaine des «lits et ameublements» couvrant plus de 800 pages d'inventaire, «il eût été impossible de publier ce chapitre en entier avec tous ses développements. De là nécessité, ou de supprimer un certain nombre d'articles, ou de retrancher les descriptions inutiles, les détails superflus » (GUIFFREY, Jules. Op. cit., vol. II, p. VIII-IX).

8. - AN, $\mathrm{O}^{1} 3332$, fo $291 \mathrm{v}^{\circ}-295$ ( $\left.\mathrm{n}^{\circ} 1380-1386\right)$.

9. - Voir également le Journal du Garde-Meuble : AN, $\mathrm{O}^{1} 3306, \mathrm{f}^{\circ} 125 \mathrm{v}^{\mathrm{o}}-135$. Les transformations commencèrent dès 1687 (ibid., 259 AP 62, fo 56-57 $\mathrm{V}^{\circ} ; 259 \mathrm{AP} 43$, fo $175-176 \mathrm{~V}^{\circ}$ ).

10. - AN, $\mathrm{O}^{1} 3282 \mathrm{~B}$.

11. - AN, $\mathrm{O}^{1} 3331$, fo $1-6$ ( $\left.\mathrm{n}^{\circ} 1-43\right), 21$ ( $\mathrm{n}^{\circ} 88-89$ ).

12. - Dans ces travaux, il faudrait pouvoir préciser le rôle de Robert I Remy, brodeur de la reine et garde de ses meubles de broderie dès 1564. D'autres brodeurs ont travaillé pour Jeanne d'Albret (RAYMOND, Paul. « Notes extraites des comptes de Jeanne d'Albret et de ses enfants, 1556-1608 ». Revue d'Aquitaine et des Pyrénées. X, 1866, p. 569, 573 et XI, 1867, p. 128).

13. - THOMAS, Danièle. Inventaires mobiliers et pièces annexes : château de Pau, château de Nérac (XVI et XVII siècles). Pau : Société des amis du château, 1996, p. 50, nº 6.

14. - LAPRADE, Jacques de. «Un inventaire des tentures et des meubles transportés de Pau à Nérac en 1578 ». Bibliothèque d'humanisme et Renaissance. Travaux et documents, XXIV, 1962, p. 418, $\mathrm{n}^{\circ} 8$, p. $422, \mathrm{n}^{\circ} 43-45$, p. $429, \mathrm{n}^{\circ} 131$.

15. - LAPRADE, Jacques de. «Richesses anciennes du château de Pau. L'envoi à Paris de la tenture et du lit des Devises en 1597 et 1601 ». Bulletin de la Société des sciences, lettres et arts de Pau, XXV, 1964 (tiré à part non paginé).

16. - LAPRADE, Jacques de, et PÉROT, Jacques. «La destinée du meuble de Pau sous Henri IV : les pièces envoyées à Fontainebleau en 1602 ». Dans Provinces et pays du Midi au temps d'Henri de Navarre (1555-1589). Actes du colloque de Bayonne, 7-9 octobre 1988. Pau: château de Pau/ Association Henri IV, 1989, p. 206-207.

17. - Ibid., p. 206-208, 217, $\mathrm{n}^{\circ} 14$.

18. - GODEFROY, Théodore et Denys. Le Cérémonial françois... Paris : Sébastien et Gabriel Cramoisy, 1649, II, p. 178 : « une tapisserie de Navarre, appellée les Devises de Pau »; RAHLENBECK, Charles. «Les tapisseries des rois de Navarre ». Messager des sciences historiques et des arts de Belgique, 1868, p. 383.

19. - GODEFROY, Théodore et Denys. Op. cit., I, p. 442 ; GUIFFREY, Jules. Histoire générale de la tapisserie. I. France. Paris : Société anonyme de publications périodiques, 1878-1885, p. 69.

20. - FAVREAU, Marc. Art. cit., p. 57-58, cite notamment le chiffre AJ (Jeanne d'Albret).

21. - AN, $\mathrm{O}^{1} 3304, \mathrm{f}^{\circ} 68 \mathrm{~V}^{\circ}$, avec une confusion possible avec la tenture $\mathrm{n}^{\circ} 3$ ci-dessous.

22. - AN, $\mathrm{O}^{1} 3306$, fo $128 \mathrm{v}^{\circ}-129,130 \mathrm{v}^{\circ}, 134 \mathrm{v}^{\circ}, 135$, cite « divers sujets de métamorphoses ».

23. - $A N, O^{1} 3339$, fo $1-2$.

24. - AN, $\mathrm{O}^{1} 3664$, dossier 9 ; ALCOUFFE, Daniel. «Les ventes de Louis XV ». Dans CHARLES, Jacques (dir.). De Versailles à Paris : le destin des collections royales. Cat. exp., Paris, centre culturel du Panthéon. Paris: centre culturel du Panthéon, 1989, p. 49 ; FAVREAU, Marc. Art. cit., p. 65, note 31.

25. - SCHNEEBALG-PERELMAN, Sophie. «Richesses du garde-meuble parisien de François $\mathrm{I}^{\mathrm{er}}$. Inventaires inédits de 1542 et $1551 »$. Gazette des beaux-arts, 1971, 78, p. 253-304, ici p. 292, no 296-301 (?) (longue description).

26. - FAVREAU, Marc. Art. cit., p. 57 (liste des sujets).

27. - GUIFFREY, Jules. Inventaire général... Op. cit., II, p. 388 ; AN, $\mathrm{O}^{1} 3306$, fo $127 \mathrm{r}^{\circ}-\mathrm{v}^{\circ}, 131,132,134$, $\mathrm{n}^{\circ} 1383$. 
28. - AN, $\mathrm{O}^{1} 3339, \mathrm{f}^{\mathrm{o}} 40 \mathrm{v}^{\mathrm{o}}-42 \mathrm{v}^{\circ}$ (longue description).

29. - FAVREAU, Marc. Art. cit., p. 65 , note 28 ; AN, $\mathrm{O}^{1} 3662$, fo 10, $15 \mathrm{v}^{\circ}, 37 \mathrm{v}^{\circ}, 47, \mathrm{n}^{\circ} 1383 / 79$.

30. - GARASSE, François. La Doctrine curieuse des beaux esprits de ce temps, ou pretendus tels... Paris : Sébastien Chappelet, 1623, p. 225-226 ; RAHLENBECK, Charles. Art. cit., p. 383-384.

31. - LAPRADE, «Un inventaire des tentures et des meubles transportés de Pau à Nérac en 1578 ». Art. cit., p. 417, $\mathrm{n}^{\circ} 1$, p. $419, \mathrm{n}^{\circ} 17$.

32. - LAPRADE, Jacques de, et PÉROT, Jacques. « La destinée du meuble de Pau sous Henri IV : les pièces envoyées à Fontainebleau en 1602 ». Art. cit., p. 216, $n^{\circ} 1$ et p. 222, nº 70, citée à Pau dès 1562.

33. - SULLY, Maximilien de Béthune, duc de. Mémoires des sages et royales œeconomies d'Estat, domestiques, politiques et militaires de Henry le Grand... Amsterdam : [1640], I, p. 503.

34. - GODEFROY, Théodore et Denys. Op. cit., I, p. 442.

35. - THOMAS, Danièle. Op. cit., p. 171, nº 40, p. 173, no 56.

36. - DUBUISSON-AUBENAY, François-Nicolas Baudot. Journal des guerres civiles, 1648-1652. Éd. Gustave Saige. Paris : H. Champion, 1883-1885, t. I, p. 262.

37. - FAVREAU, Marc. Art. cit., p. 58 (liste des sujets).

38. - AN, $\mathrm{O}^{1} 3304, \mathrm{f}^{\circ} 5 \mathrm{v}^{\circ}, 48 \mathrm{v}^{\circ}, 133$.

39. - GUIFFREY, Jules. Inventaire général... Op. cit., t. II, p. 388-389; AN, $\mathrm{O}^{1} 3306$, fo $127 \mathrm{v}^{\mathrm{o}}-128,131$, $134 \mathrm{v}^{\circ}, \mathrm{n}^{\circ} 1384$.

40. - AN, $\mathrm{O}^{1} 3339$, fo $42 \mathrm{v}^{\circ}$.

41. - FAVREAU, Marc. Art. cit., p. 65 , note 33 ; AN, $\mathrm{O}^{1} 3662$, fo $37 \mathrm{v}^{\circ}, 46 \mathrm{v}^{\circ}, \mathrm{n}^{\circ} 1384 / 80$.

42. - SCHNEEBALG-PERELMAN, Sophie. Art. cit., p. 274-275, $\mathrm{n}^{\circ}$ 44-49 (longue description).

43. - GUIFFREY, Jules. Inventaire général... Op. cit., t. II, p. 387 ; AN, $\mathrm{O}^{1} 3306, \mathrm{f}^{\circ} 126 \mathrm{~V}^{\mathrm{o}}, 131,134, \mathrm{n}^{\circ}$ 1381 et 1382 .

44. - $\mathrm{AN}, \mathrm{O}^{1} 3339, \mathrm{f}^{\mathrm{o}} 40 \mathrm{r}^{\circ}-\mathrm{V}^{\mathrm{o}}$.

45. - AN, $\mathrm{O}^{1} 3662$, fo $37 \mathrm{r}^{\mathrm{o}}-\mathrm{v}^{\mathrm{o}}, 46 \mathrm{v}^{\mathrm{o}}, \mathrm{n}^{\circ} 1381 / 77,1382 / 78$.

46. - THOMAS, Danièle. Op. cit., p. $21, \mathrm{n}^{\circ} 1$.

47. - LAPRADE, Jacques de. «Un inventaire des tentures et des meubles transportés de Pau à Nérac en 1578 ». Art. cit., p. 417, nº 4 .

48. - LAPRADE, Jacques de, et PÉROT, Jacques. « La destinée du meuble de Pau sous Henri IV : les pièces envoyées à Fontainebleau en 1602 ». Art. cit., p. 222, nº 72.

49. - GODEFROY, Théodore et Denys. Op. cit., I, p. 442.

50. - FAVREAU, Marc. Art. cit., p. 57 (liste des sujets).

51. - GUIFFREY, Jules. Inventaire général... Op. cit., t. II, p. 389 ; AN, $\mathrm{O}^{1} 3306$, fo $128,131 \mathrm{v}^{\circ}, 134, \mathrm{n}^{\circ}$ 1385.

52. - AN, $\mathrm{O}^{1} 3339$, fo $42 \mathrm{v}^{\mathrm{o}}-43$.

53. - AN, $\mathrm{O}^{1} 3664$, dossier 10 ; FAVREAU, Marc. Art. cit., p. 65, note 27.

54. - AN, $\mathrm{O}^{1} 3662, \mathrm{f}^{\circ} 37 \mathrm{v}^{\mathrm{o}}, 46 \mathrm{v}^{\circ}, \mathrm{n}^{\circ} 1385 / 81$.

55. - LAPRADE, Jacques de. «Un inventaire des tentures et des meubles transportés de Pau à Nérac en 1578 ». Art. cit., p. 417, $\mathrm{n}^{\circ} 2$.

56. - LAPRADE, Jacques de, et PÉROT, Jacques. « La destinée du meuble de Pau sous Henri IV : les pièces envoyées à Fontainebleau en 1602 ». Art. cit., p. 216, nº 2, p. 222, nº 71.

57. - GODEFROY, Théodore et Denys. Op. cit., I, p. 442.

58. - FAVREAU, Marc. Art. cit., p. 58 (liste des sujets).

59. - GUIFFREY, Jules. Inventaire général... Op. cit., t. II, p. 389 ; AN, $\mathrm{O}^{1} 3306, \mathrm{f}^{\circ} 128 \mathrm{r}^{\circ}-\mathrm{v}^{\circ}, 129,130 \mathrm{r}^{\mathrm{o}}-\mathrm{v}^{\mathrm{o}}$, $131 \mathrm{v}^{\circ}-132,133 \mathrm{v}^{\circ}, 134 \mathrm{v}^{\mathrm{o}}-135, \mathrm{n}^{\circ} 1386$.

60. - AN, $\mathrm{O}^{1} 3339, \mathrm{f}^{\circ} 2,43 \mathrm{r}^{\circ}-\mathrm{v}^{\mathrm{o}}$.

61. - AN, $\mathrm{O}^{1} 3664$, dossier 10 ; ALCOUFFE, Daniel. Art. cit., p. 49 ; FAVREAU, Marc. Art. cit., p. 65, note 38 .

62. - AN, $\mathrm{O}^{1} 3662, \mathrm{f}^{\circ} 37 \mathrm{v}^{\circ}, 46 \mathrm{v}^{\circ}-47, \mathrm{n}^{\circ} 1386 / 82$. 
63. - RAHLENBECK, Charles. Art. cit., p. 386 ; LAPRADE, Jacques de. « Un inventaire des tentures et des meubles transportés de Pau à Nérac en 1578 ». Art. cit., p. 418, nº 9-16.

64. - LAPRADE, Jacques de, et PÉROT, Jacques. « La destinée du meuble de Pau sous Henri IV : les pièces envoyées à Fontainebleau en 1602 ». Art. cit., p. 218, nº 20-21, p. 221, n 55, 58, p. 223, nº 73, 78.

65. - FAVREAU, Marc. Art. cit., p. 57, bien décrit.

66. - $\mathrm{AN}, \mathrm{O}^{1} 3339$, fo $2 \mathrm{r}^{\mathrm{o}}-\mathrm{V}^{\mathrm{o}}$.

67. - $\mathrm{AN}, \mathrm{O}^{1} 3339, \mathrm{f}^{\circ} 2 \mathrm{v}^{\mathrm{o}}-3 \mathrm{v}^{\mathrm{o}}$.

68. - AN, $\mathrm{O}^{1} 3664$, dossier 9, avec le dais $\mathrm{n}^{\circ} 15$; ALCOUFFE, Daniel. Art. cit., p. 49.

69. - HAMY, Alfred. Entrevue de François Premier avec Henry VIII à Boulogne-sur-Mer en 1532. Intervention de la France dans l'affaire du divorce, d'après un grand nombre de documents inédits. Paris : L. Gougy, 1898, p. 81, CXXV.

70. - AN, $\mathrm{O}^{1} 3339$, fo $3 \mathrm{v}^{\mathrm{o}}-4$.

71. - AN, $\mathrm{O}^{1} 3664$, dossier 5 , pièces 7 et 8 , et dossier 7 .

72. - SCHNEEBALG-PERELMAN, Sophie. Art. cit., p. 296, $\mathrm{n}^{\circ}$ 380-385.

73. - GUIFFREY, Jules. Inventaire général... Op. cit., t. II, p. 386-387; AN, $\mathrm{O}^{1} 3306$, fo $125 \mathrm{v}^{\circ}-126 \mathrm{v}^{\circ}$, $129 \mathrm{v}^{\circ}, 132, \mathrm{n}^{\circ} 1380$.

74. - AN, $\mathrm{O}^{1} 3339$, fo $38-40$.

75. - AN, $\mathrm{O}^{1} 3662, \mathrm{f}^{\mathrm{o}} 1 \mathrm{v}^{\mathrm{o}}, 10,14,15 \mathrm{v}^{\mathrm{o}}, \mathrm{n}^{\circ} 1380 / 76$.

76. - GUIFFREY, Jules. Inventaire général... Op. cit., t. II, p. 386-387; AN, $\mathrm{O}^{1} 3306, \mathrm{f}^{\circ} 125 \mathrm{v}^{\circ}-126 \mathrm{v}^{\mathrm{o}}, 130$, $132,134, \mathrm{n}^{\circ} 1380$.

77. - $\mathrm{AN}, \mathrm{O}^{1} 3339$, fo $38-40$.

78. - AN, $\mathrm{O}^{1} 3662$, fo $10,15 \mathrm{v}^{\circ}, \mathrm{n}^{\circ} 11 ; \mathrm{O}^{1} 3665$, dossier 3 .

79. - $\mathrm{AN}, \mathrm{O}^{1} 3306, \mathrm{f}^{\circ} 129 \mathrm{v}^{\circ}, 130,132 \mathrm{v}^{\circ}$.

80. - $A N, O^{1} 3662, f^{\circ} 10,15 v^{\circ}, n^{\circ} 12$.

81. - LAPRADE, Jacques de, et PÉROT, Jacques. « La destinée du meuble de Pau sous Henri IV : les pièces envoyées à Fontainebleau en 1602 ». Art. cit., p. 217, nº 7.

82. - $\mathrm{AN}, \mathrm{O}^{1} 3339$, fo 4 .

83. - $\mathrm{AN}, \mathrm{O}^{1} 3339$, fo 4 .

84. - LAPRADE, Jacques de. "Un inventaire des tentures et des meubles transportés de Pau à Nérac en $1578 »$. Art. cit., p. 421, nº 36, p. 424, nº 72, p. 429, nº 132.

85. - LAPRADE, Jacques de, et PÉROT, Jacques. « La destinée du meuble de Pau sous Henri IV : les pièces envoyées à Fontainebleau en 1602 ». Art. cit., p. 217, nº 11, p. 219, nº 38.

86. - $\mathrm{AN}, \mathrm{O}^{1} 3306, \mathrm{f}^{\circ} 129 \mathrm{v}^{0}, 132 \mathrm{v}^{\mathrm{o}}-133$.

87. - AN, $\mathrm{O}^{1} 3339, \mathrm{f}^{\mathrm{o}} 4 \mathrm{r}^{\mathrm{o}}-\mathrm{v}^{\mathrm{o}}$

88. - AN, $\mathrm{O}^{1} 3664$, dossier 5 , pièces 7 et 8 , et dossier 7 .

89. - LEVI, Honor. «L'inventaire après décès du cardinal de Richelieu ». Archives de l'Art français, XXVII, 1985, p. 34, n 418 .

90. - Ibid., p. $76, \mathrm{n}^{\circ} 418$.

91. - COSNAC, Gabriel-Jules de. Mazarin et Colbert. Paris : E. Plon-Nourrit, 1892, t. II, p. 383, 398.

92. - LABORDE, Léon de. La Renaissance des arts à la cour de France. Études sur le seizième siècle. Paris : L. Potier, 1850-1855, II, p. 1000.

93. - AN, $\mathrm{O}^{1} 3339$, fo $4-6$.

94. - LABORDE, Léon de. Op.cit., p. 993-1003, description complète, la commande attribuée à François I ${ }^{\mathrm{er}}$.

95. - AN, $\mathrm{O}^{1} 3662, \mathrm{f}^{\circ} 10,15 \mathrm{v}^{\circ}-16, \mathrm{n}^{\circ} 40 ; \mathrm{O}^{1} 3665$, dossier 3.

96. - PRIVAT-SAVIGNY, Marie-Anne, et BARBIER, Muriel, dans CRÉPIN-LEBLOND, Thierry et THIRION, Jacques (dir.). Parures d'or et de pourpre. Le mobilier à la cour des Valois. Cat. exp., château de Blois, 15 juin-30 septembre 2002. Paris/Blois : château de Blois/Somogy éditions d'art, 2002, notices $n^{\circ} 9$ et 3 . 
97. - $\mathrm{AN}, \mathrm{O}^{1} 3339, \mathrm{f}^{\mathrm{o}} 6 \mathrm{~V}^{\mathrm{o}}-7$.

98. - VÉRON-DENISE, Danièle. «Notes sur un lit brodé de Mazarin, dans lequel la reine Anne d'Autriche accoucha du dauphin, futur Louis XIV ». Dans CONIHOUT, Isabelle de, et MICHEL, Patrick. Mazarin, les Lettres et les Arts. Saint-Rémy-en-l'Eau et Paris : 2006.

99. - BnF, Mélanges Colbert $270, \mathrm{f}^{\circ} 11 \mathrm{r}^{\circ}-\mathrm{v}^{\circ}$.

100. - AN, $\mathrm{O}^{1} 3662$, fo $10,15 \mathrm{~V}^{\circ}, \mathrm{n}^{\circ} 88 / 36$.

101. - VÉRON-DENISE, Danièle. « Un brodeur de Henri II : Robert Mestays ». Dans OURSEL, Hervé, et FRITSCH, Julia. Henri II et les Arts. Actes du colloque international organisé par l'École du Louvre et le musée national de la Renaissance-Écouen, 25, 26 et 27 septembre 1997. Paris : École du Louvre, 2003.

102. - DEVILLE, Jules. Dictionnaire du tapissier critique et historique de l'ameublement français depuis les temps anciens jusqu'à nos jours. Paris/Liège/Berlin : s.n., 1878-1880, p. 303.

103. - VÉRON-DENISE, Danièle. « Un brodeur de Henri II : Robert Mestays ». Art. cit.

104. - BnF, Mélanges Colbert $270, \mathrm{f}^{\circ} 11 \mathrm{r}^{\circ}-\mathrm{v}^{\circ}$.

105. - AN, $\mathrm{O}^{1} 3339$, fo $16 \mathrm{v}^{\mathrm{o}}-17 \mathrm{v}^{\mathrm{o}}$.

106. - AN, $\mathrm{O}^{1} 3662$, fo $10,15 \mathrm{v}^{\circ}, \mathrm{n}^{\circ} 89 / 37$.

107. - CRÉPIN-LEBLOND, Thierry et THIRION, Jacques (dir.). Parures d'or et de pourpre. Op. cit., $\mathrm{n}^{\circ}$ 17 ; CASTRES, Astrid. Dans Henri II à Saint-Germain-en-Laye. Une cour royale à la Renaissance. Cat. exp., Saint-Germain-en-Laye, musée des Antiquités nationales, 31 mars-14 juillet 2019. Paris : RMN-GP, 2019, n 34-35.

108. - AN, $O^{1} 3331$, fo $137-140\left(n^{\circ} 1-34\right)$.

109. - $\mathrm{AN}, \mathrm{O}^{1} 3338, \mathrm{f}^{\mathrm{o}} 1$; les dimensions y sont précisées, comme pour tous les dais.

110. - AN, $O^{1} 3664$, dossiers 6 et 7 .

111. - AN, $\mathrm{O}^{1} 3659$, fo $192, \mathrm{n}^{\circ} 394$.

112. - AN, $\mathrm{O}^{1} 3306, \mathrm{f}^{\circ} 133$.

113. - $A N, O^{1} 3338$, fo 1 .

114. - AN, $\mathrm{O}^{1} 3664$, dossier 9.

115. - THOMAS, Danièle. Op. cit., p. 21, $\mathrm{n}^{\circ} 11$, p. 50, no 12, p. $62, \mathrm{n}^{\circ} 20$.

116. - LAPRADE, Jacques de. "Un inventaire des tentures et des meubles transportés de Pau à Nérac en 1578 ». Art. cit., p. 417, nº 3 (?), p. 419, nº 23 ou 24, p. 422, nº 41 (?).

117. - THOMAS, Danièle. Op. cit., p. 85, $\mathrm{n}^{\circ} 108$.

118. - LAPRADE, Jacques de, et PÉROT, Jacques. « La destinée du meuble de Pau sous Henri IV : les pièces envoyées à Fontainebleau en 1602 ». Art. cit., p. 216, nº 5.

119. - $\mathrm{AN}, \mathrm{O}^{1} 3338, \mathrm{f}^{\circ} 1 \mathrm{r}^{\mathrm{o}}-\mathrm{v}^{\circ}$.

120. - AN, $\mathrm{O}^{1} 3664$, dossiers 6 et 7 .

121. - AN, $\mathrm{O}^{1} 3659$, fo $11 \mathrm{v}^{\circ}-12, \mathrm{n}^{\circ} 27$.

122. - $A N, O^{1} 3338, f^{\circ} 1 v^{0}-2$.

123. - AN, $\mathrm{O}^{1} 3664$, dossier 10 .

124. - HAMY, Alfred. Op. cit., p. 50, XXXIII.

125. - SCHNEEBALG-PERELMAN, Sophie. Art. cit., p. 276, $\mathrm{n}^{\circ}$ 57-58.

126. - $\mathrm{AN}, \mathrm{O}^{1} 3338, \mathrm{f}^{\circ} 2$.

127. - AN, $\mathrm{O}^{1} 3662, \mathrm{f}^{\circ} 10,21 \mathrm{v}^{\circ}, 70 \mathrm{v}^{\circ}, 76, \mathrm{n}^{\circ} 5$.

128. - LAPRADE, Jacques de. «Un inventaire des tentures et des meubles transportés de Pau à Nérac en $1578 »$. Art. cit., p. 419, $\mathrm{n}^{\circ} 18$.

129. - LAPRADE, Jacques de, et PÉROT, Jacques. « La destinée du meuble de Pau sous Henri IV : les pièces envoyées à Fontainebleau en 1602 ». Art. cit., p. 216, $\mathrm{n}^{\circ} 2$.

130. - AN, $\mathrm{O}^{1} 3306, \mathrm{f}^{\circ} 130 \mathrm{r}^{\circ}-\mathrm{v}^{\circ}, 131 \mathrm{v}^{\circ}, 133 \mathrm{v}^{\circ}, 134 \mathrm{v}^{\mathrm{o}}-135$.

131. - AN, $\mathrm{O}^{1} 3338$, fo 2 .

132. - AN, $\mathrm{O}^{1} 3664$, dossier 10 . 
133. - LAPRADE, Jacques de. "Au temps de Marguerite d'Angoulême, un inventaire mobilier de Nérac (1552) ». Le Vieux Papier, XXIII, 1961-1963, p. 261, nº 16.

134. - THOMAS, Danièle. Op. cit., p. 50, $\mathrm{n}^{\circ} 11$.

135. - Ibid., p. 62, $\mathrm{n}^{\circ} 21$.

136. - LAPRADE, Jacques de. "Un inventaire des tentures et des meubles transportés de Pau à Nérac en $1578 »$. Art. cit., p. 419, nº 20.

137. - LAPRADE, Jacques de, et PÉROT, Jacques. « La destinée du meuble de Pau sous Henri IV : les pièces envoyées à Fontainebleau en $1602 »$. Art. cit., p. 220, nº 45.

138. - $\mathrm{AN}, \mathrm{O}^{1} 3338, \mathrm{f}^{\mathrm{o}} 2 \mathrm{r}^{\mathrm{o}}-\mathrm{v}^{\mathrm{o}}$.

139. - AN, $\mathrm{O}^{1} 3664$, dossiers 6 et 7.

140. - AN, $O^{1} 3659$, fo $238 v^{\circ}-239, n^{\circ} 493$.

141. - $\mathrm{AN}, \mathrm{O}^{1} 3338$, fo $2 \mathrm{v}^{\circ}$.

142. - $\mathrm{AN}, \mathrm{O}^{1} 3664$, dossier 5 , pièces 7 et 8 , et dossier 7 .

143. - LAPRADE, Jacques de, et PÉROT, Jacques. «La destinée du meuble de Pau sous Henri IV : les pièces envoyées à Fontainebleau en 1602 ». Art. cit., p. 221, nº 55.

144. - $\mathrm{AN}, \mathrm{O}^{1} 3338$, fo $2 \mathrm{v}^{\mathrm{o}}-3$.

145. - AN, $\mathrm{O}^{1} 3664$, dossier 9.

146. - LAPRADE, Jacques de. «Au temps de Marguerite d'Angoulême, un inventaire mobilier de Nérac (1552) ». Art. cit., p. 260, nº 1, p. 264, nº 98 (tenture), p. 265, n 101-103.

147. - THOMAS, Danièle. Op. cit., p. $62, \mathrm{n}^{\circ} 12$.

148. - LAPRADE, Jacques de. «Un inventaire des tentures et des meubles transportés de Pau à Nérac en $1578 »$. Art. cit., p. 419, n 19, p. 427, nº 102.

149. - LAPRADE, Jacques de, et PÉROT, Jacques. « La destinée du meuble de Pau sous Henri IV : les pièces envoyées à Fontainebleau en 1602 ». Art. cit., p. 220, nº 44.

150. - THOMAS, Danièle. Op. cit., p. $168, \mathrm{n}^{\circ} 18$.

151. - GUIFFREY, Jules. Inventaire général... Op. cit., t. II, p. 386-387; AN, $\mathrm{O}^{1} 3306, \mathrm{f}^{\circ} 125 \mathrm{v}^{\mathrm{o}}-126 \mathrm{v}^{\circ}$, $133 \mathrm{v}^{\circ}, \mathrm{n}^{\circ} 1380$.

152. - AN, $\mathrm{O}^{1} 3338, \mathrm{f}^{\circ} 3 ; \mathrm{O}^{1} 3339$, fo $38-40, \mathrm{n}^{\circ} 52$.

153. - LAPRADE, 1961-1963, p. 260, $\mathrm{n}^{\circ} 5$.

154. - THOMAS, Danièle. Op. cit., p. 50, $\mathrm{n}^{\circ} 10$, p. 62, $\mathrm{n}^{\circ} 15$.

155. - RAHLENBECK, Charles. Art. cit., p. 387 ; LAPRADE, Jacques de. «Un inventaire des tentures et des meubles transportés de Pau à Nérac en 1578 ». Art. cit., p. 418, $\mathrm{n}^{\circ} 7$ (tenture), p. 420, $\mathrm{n}^{\circ} 31$, p. $423, \mathrm{n}^{\circ} 51$ (tenture).

156. - LAPRADE, Jacques de, et PÉROT, Jacques. « La destinée du meuble de Pau sous Henri IV : les pièces envoyées à Fontainebleau en 1602 ». Art. cit., p. 221, nº 52.

157. - THOMAS, Danièle. Op. cit., p. 176, $\mathrm{n}^{\circ} 72$.

158. - AN, $\mathrm{O}^{1} 3338, \mathrm{f}^{\mathrm{o}} 3$.

159. - AN, $\mathrm{O}^{1} 3664$, dossier 5 , pièces 7 et 8 , et dossier 7 .

160. - LAPRADE, Jacques de. «Un inventaire des tentures et des meubles transportés de Pau à Nérac en 1578 ». Art. cit., p. 421, nº 37.

161. - LAPRADE, Jacques de, et PÉROT, Jacques. « La destinée du meuble de Pau sous Henri IV : les pièces envoyées à Fontainebleau en 1602 ». Art. cit., p. 221, nº 53.

162. - GUIFFREY, Jules. Inventaire général... Op. cit., t. II, p. 386-387; AN, $\mathrm{O}^{1} 3306$, fo $125 \mathrm{v}^{\circ}-126 \mathrm{v}^{\circ}$, $133 \mathrm{v}^{\circ}, 134, \mathrm{n}^{\circ} 1380$.

163. - AN, $\mathrm{O}^{1} 3338$, fo $3 ; \mathrm{O}^{1} 3339$, fo $38-40, \mathrm{n}^{\circ} 52$.

164. - AN, $\mathrm{O}^{1} 3664$, dossier 9.

165. - AN, $\mathrm{O}^{1} 3662$, fo $1 \mathrm{v}^{\circ}, \mathrm{n}^{\circ} 1380$.

166. - LAPRADE, Jacques de. «Au temps de Marguerite d'Angoulême, un inventaire mobilier de Nérac (1552)». Art. cit., p. 260, nº 2 . 
167. - LAPRADE, Jacques de. «Un inventaire des tentures et des meubles transportés de Pau à Nérac en $1578 »$. Art. cit., p. 422, $\mathrm{n}^{\circ} 42$.

168. - LAPRADE, Jacques de, et PÉROT, Jacques. «La destinée du meuble de Pau sous Henri IV : les pièces envoyées à Fontainebleau en 1602 ». Art. cit., p. 221, nº 54.

169. - $\mathrm{AN}, \mathrm{O}^{1} 3338, \mathrm{f}^{\mathrm{o}} 3 \mathrm{v}^{\circ}$.

170. - AN, $\mathrm{O}^{1} 3664$, dossiers 6 et 7 .

171. - AN, $O^{1} 3659$, fo $161 v^{\circ}-162, n^{\circ} 329$.

172. - THOMAS, Danièle. Op. cit., p. $50, \mathrm{n}^{\circ} 11$.

173. - $\mathrm{AN}, \mathrm{O}^{1} 3338, \mathrm{f}^{\mathrm{O}} 3 \mathrm{v}^{\circ}$.

174. - $\mathrm{AN}, \mathrm{O}^{1} 3338$, fo 4 .

175. - $\mathrm{AN}, \mathrm{O}^{1} 3338, \mathrm{f}^{\circ} 4$.

176. - AN, $\mathrm{O}^{1} 3338, \mathrm{f}^{\mathrm{O}} 4 \mathrm{v}^{\mathrm{O}}$.

177. - AN, $\mathrm{O}^{1} 3664$, dossier 9.

178. - $A N, O^{1} 3338, f^{\circ} 4 v^{\circ}$.

179. - AN, $\mathrm{O}^{1} 3664$, dossiers 6 et 7 .

180. - AN, $\mathrm{O}^{1} 3659$, fo $271 \mathrm{v}^{\circ}, \mathrm{n}^{\circ} 561$.

181. - AN, $\mathrm{O}^{1} 3338$, fo $4 \mathrm{v}^{\mathrm{o}}-5 \mathrm{v}^{\circ}$.

182. - AN, $\mathrm{O}^{1} 3662$, fo $10,21 v^{\circ}, \mathrm{n}^{\circ} 19$.

183. - $\mathrm{AN}, \mathrm{O}^{1} 3338, \mathrm{f}^{\mathrm{O}} 5 \mathrm{v}^{\mathrm{0}}-6$.

184. - AN, $\mathrm{O}^{1} 3662$, fo $42 \mathrm{v}^{\circ}, 52 \mathrm{v}^{\circ}, \mathrm{n}^{\circ} 22$.

185. - AN, $\mathrm{O}^{1} 3338, \mathrm{f}^{\circ} 6 \mathrm{r}^{\circ}-\mathrm{v}^{\mathrm{o}}$.

186. - AN, $\mathrm{O}^{1} 3664$, dossier 9 .

187. - GUIFFREY, Jules. « Inventaires des meubles précieux de l'hôtel de Guise en 1644 et en 1688 et de l'hôtel de Soubise en 1787 ». Nouvelles Archives de l'art français, 1896, p. 175, nº 43.

188. - ALFASSA, Paul. "L'achat par Louis XIV des tapisseries des "Chasses de l'empereur Maximilien” ». Bulletin de la Société de l'histoire de l'art français, 1918-1919, p. 132-133, 139.

189. - AN, $\mathrm{O}^{1} 3338, \mathrm{f}^{\circ} 7$.

190. - $A N, O^{1} 3662$, fo $10 v^{\circ}, 24 v^{\circ}, n^{\circ} 26$.

191. - GUIFFREY, Jules. Art. cit., p. 175, $\mathrm{n}^{\circ} 44$.

192. - ALFASSA, Paul. Art. cit., p. 132-133, 139.

193. - $\mathrm{AN}, \mathrm{O}^{1} 3338, \mathrm{f}^{\circ} 7 \mathrm{r}^{\mathrm{o}}-\mathrm{v}^{\circ}$.

194. - AN, $\mathrm{O}^{1} 3662$, fo $10 \mathrm{v}^{\circ}, 24 \mathrm{v}^{\circ}, \mathrm{n}^{\circ} 27$.

195. - AN, $O^{1} 3338$, fo 9 .

196. - AN, $O^{1} 3664$, dossier 5 , pièces 7 et 8 , et dossier 7 .

197. - LAPRADE, Jacques de. "Un inventaire des tentures et des meubles transportés de Pau à Nérac en 1578 ». Art. cit., p. 420, n 26.

198. - LAPRADE, Jacques de, et PÉROT, Jacques. «La destinée du meuble de Pau sous Henri IV : les pièces envoyées à Fontainebleau en 1602 ». Art. cit., p. 220, nº 48.

199. - $A N, O^{1} 3338$, fo $9 \mathrm{r}^{\circ}-\mathrm{v}^{\circ}$.

200. - AN, $O^{1} 3662$, fo $7,21 v^{\circ}, n^{\circ} 34$. 


\section{RÉSUMÉS}

En matière de lits, les collections royales françaises conservaient à la Renaissance des œuvres exceptionnelles, mais, en raison de leur disparition au XVIII ${ }^{\mathrm{e}}$ siècle et du caractère lacunaire des comptes et des inventaires, il est souvent difficile d'en avoir une connaissance précise. Pourtant les documents descriptifs rédigés postérieurement, lorsque les œuvres existaient encore, peuvent nous aider notablement à préciser leur apparence. De ce point de vue, l'Inventaire général du mobilier de la Couronne sous Louis XIV (1673), publié par Jules Guiffrey à la fin du XIXe siècle, qui décrit un certain nombre de lits ou de dais de la Renaissance, s'avère d'une grande utilité. L'article est suivi de la publication révisée des descriptions des lits, accompagnées de l'historique de ceux-ci tel qu'il peut être reconstitué à l'aide des inventaires et des archives du Garde-Meuble.

Where beds are concerned, the French royal collections held some exceptional works at the time of the Renaissance, but because of their subsequent disappearance, during the eighteenth century, and because of deficiencies in the archival accounts and inventories, it is often difficult to have precise knowledge of them. However, descriptive documents written later, when the beds were still in existence, can help considerably in giving us an idea of what these beds looked like. The general inventory of crown furniture under Louis XIV, drawn up in 1673 and published by Jules Guiffrey at the end of the nineteenth century, describes a certain number of Renaissance beds or canopies and is of particular usefulness. The present article is followed by a revised publication of the bed descriptions, along with the history of the beds as it can be reconstructed using the inventories and archives of the crown's furniture repository, the Garde-Meuble.

\section{INDEX}

Mots-clés : Alençon, Amboise, Ancien Testament, Anne d'Autriche, Bourbons, Claude de France, dais, Diane de Poitiers, ducs de Guise, François Ier, Gabrielle d'Estrées, Garde-Meuble, Hélène, Henri II, Henri IV, Henry VIII, inventaire général du mobilier de la Couronne, Jeanne d'Albret, Joseph, Judith, Jules Romain, lits, Louis XIII, Louis XIV, Marie de Médicis, Mazarin, Mélusine, métamorphoses, Moïse, Nérac, Michel Particelli d'Emery, Pau, Raphaël, Richelieu, rois de Navarre, Saint-Germain-en-Laye, salamandre, satyres, Valois, Versailles, Vincennes

Keywords : Alençon, Amboise, Old Testament, Anne of Austria, Bourbons, Claude de France, canopy, Diane de Poitiers, Dukes de Guise, François Ier, Gabrielle d'Estrées, royal furniture repository (Garde-Meuble), Hélène, Henri II, Henri IV, Henry VIII, general inventory of crown furniture, Jeanne d'Albret, Joseph, Judith, Jules Romain, beds, Louis XIII, Louis XIV, Marie de Médicis, Mazarin, Mélusine, metamorphosis, Moïse, Nérac, Michel Particelli d'Emery, Pau, Raphaël, Richelieu, kings of Navarre, Saint-Germain-en-Laye, salamander, satyrs, Valois, Versailles, Vincennes

\section{AUTEUR}

\section{JEAN VITTET}

Conservateur en chef du patrimoine, chargé du mobilier historique et des arts décoratifs avant 1815, château de Fontainebleau jean.vittet@chateaudefontainebleau.fr 